\title{
Polarization of a stochastic gravitational wave background through diffusion by massive structures
}

\author{
Giulia Cusin, ${ }^{1}$ Ruth Durrer, ${ }^{2}$ and Pedro G. Ferreira ${ }^{1}$ \\ ${ }^{1}$ Astrophysics Department, University of Oxford, DWB, Keble Road, Oxford OX1 3RH, United Kingdom \\ ${ }^{2}$ Départment de Physique Théorique and Center for Astroparticle Physics, Université de Genève,
} Quai E. Ansermet 24, CH-1211 Genève 4, Switzerland

(Received 30 August 2018; published 30 January 2019)

\begin{abstract}
The geometric optics approximation traditionally used to study the propagation of gravitational waves on a curved background, breaks down in the vicinity of compact and extended astrophysical objects, where wavelike effects like diffusion and generation of polarization occur. We provide a framework to study the generation of polarization of a stochastic background of gravitational waves propagating in an inhomogeneous universe. The framework is general and can be applied to both cosmological and astrophysical gravitational wave backgrounds in any frequency range. We derive an order of magnitude estimate of the amount of polarization generated for cosmological and astrophysical backgrounds, in the frequency range covered by present and planned gravitational wave experiments. For an astrophysical background in the PTA and LISA band, the amount of polarization generated is suppressed by a factor $10^{-4}$ $\left(10^{-5}\right)$ with respect to anisotropies. For a cosmological background we get an additional $10^{-2}$ suppression. We speculate on using our approach to map the distribution of (unresolvable) structures in the Universe.
\end{abstract}

DOI: 10.1103/PhysRevD.99.023534

\section{INTRODUCTION}

Several diffuse stochastic backgrounds of different types of radiation, arising from the incoherent superposition of signals from resolved and unresolved sources, have been observed in astronomy. In particular, backgrounds of electromagnetic radiation include the cosmic microwave background (CMB) with its black body spectrum [1], cosmic infrared background (CIB) from stellar dust [2] and the extragalactic background light made up of all the electromagnetic radiation emitted by stars, galaxies, galaxy clusters etc., since their formation [3,4]. Similarly, there should exist a neutrino background [5] and a background of gravitational waves (GW).

We can distinguish between a stochastic background of gravitational radiation of cosmological origin (CGWB) and one of astrophysical origin (AGWB). In the standard cosmological model [6], the existence of a primordial GW background from the amplification of vacuum quantum fluctuations is a generic prediction of any inflationary phase. Gravitational waves may also be produced at the end of inflation during the reheating phase (see e.g., Ref. [7] for an analytic and numerical study). More speculative sources of a GW background produced at early times include pre big-bang models, cosmic strings [8-12], first order phase transitions in the early universe $[13,14]$, magnetic fields [15]; see Refs. [16,17] for a review on those topics and Refs. [18,19] for more broader introductions. In addition, an astrophysical background results from the superposition of a large number of resolved and unresolved sources from the onset of stellar activity until today. The nature of the AGWB may differ from its cosmological counterpart, which is expected to be (roughly) stationary, unpolarized, statistically Gaussian and isotropic, by analogy with the cosmic microwave background. Many different astrophysical sources may contribute to the AGWB, including black holes and neutron star mergers [20-26], supermassive black holes [27], neutron stars [28-30], stellar core collapse 31,32]] and population III binaries [33].

The recent detection by the Advanced Laser Interferometric Gravitational-wave Observatory (LIGO) of the gravitational wave sources GW150914 [34] provided the first observation of the merging of a binary black hole system. Over the last three years, in total, nine detections and one sub-threshold candidate from binary black hole merger events have been reported, see the recent catalogue [35] for a summary. Following these observations, the rate and mass of coalescing binary black holes appear to be greater than many previous expectations. Moreover, the LIGO and Virgo ${ }^{1}$ collaboration very recently detected a new gravitational-wave source, GW170817: the coalescence of two neutron stars [36]. The merger rate of binary neutron stars estimated from this event suggests that distant binary neutron stars create a significant contribution to the AGWB which will add to the background from binary black holes,

\footnotetext{
${ }^{1}$ https://www.ego-gw.it/public/about/whatIs.aspx.
} 
increasing the amplitude of the total astrophysical background relative to previous expectations. In the Advanced LIGO-Virgo frequency band most sensitive to stochastic backgrounds (near $25 \mathrm{~Hz}$ ), the predicted amplitude of the total background is $\Omega_{\mathrm{GW}}(f=25 \mathrm{~Hz})=1.8_{-1.3}^{+2.7} \times 10^{-9}$ with $90 \%$ confidence level compared to $\Omega_{\mathrm{GW}}(f=25 \mathrm{~Hz})=$ $1.1_{-0.7}^{+1.2} \times 10^{-9}$ from binary black holes alone. Assuming the most probable rate for compact binary mergers, in [37] they find that the total background may be detectable with a signal-to-noise-ratio of 3 after 40 months of total observation time. This improves bounds on the stochastic background obtained from the analysis of big-bang nucleosynthesis [18,38], and of the cosmic microwave background $[39,40]$ at $100 \mathrm{~Hz}$. At low frequencies, pulsar timing arrays (see below) give a bound $\Omega_{\mathrm{GW}}<1.3 \times 10^{-9}$ for $f=2.8 \times$ $10^{-9} \mathrm{~Hz}$ [41]. The possibility of measuring and mapping the gravitational wave background is discussed in Refs. [42-47] while different methods employed by LIGO and LISA (Laser Interferometer Space Antenna) to reconstruct an angular resolved map of the sky are presented in Ref. [48]. An analogous discussion for pulsar timing arrays can be found in Refs. [49-51].

The observational landscape is growing and covers large bands of frequencies; see e.g., Ref. [52] for a review. ${ }^{2}$ At extremely low frequencies $\sim 10^{-16} \mathrm{~Hz}$ bounds come mainly from the analysis of CMB B-modes while at low frequency of order $10^{-10}-10^{-6} \mathrm{~Hz}$, there are pulsar timing arrays such as the radio telescope Parks Pulsar Timing Array $^{3}$ (PPTA), the Large European Array for Pulsar Timing ${ }^{4}$ (LEPTA), the future International Pulsar Timing Array ${ }^{5}$ (IPTA), the Square Kilometre Array (SKA) and the North American Nanohertz Observatory for Gravitational Waves (NANOGrav). At low frequencies (typically $10^{-6}-1 \mathrm{~Hz}$ ) detection relies on space-borne detectors, such as the Laser Interferometer Space Antenna ${ }^{6}$ (LISA) and the evolved Laser Interferometer Space Antenna ${ }^{7}$ (eLISA) selected by ESA to be launched around 2030. High frequency (typically $1-10^{5} \mathrm{~Hz}$ ) observations rely on ground-based detectors, such as LIGO and its advanced configuration (aLIGO), Virgo, the Einstein Telescope ${ }^{8}$ (ET) or its American counterpart, the Cosmic Explorer (CE) [53]. This spectrum covers most of the theoretical predictions.

Traditionally the energy density of GW of an astrophysical background has been modeled and parametrized under the assumption that both our universe and the

\footnotetext{
${ }^{2}$ The associated code http://rhcole.com/apps/GWplotter/ allows one to generate plots of noise curves for many detectors and associated target sources.

${ }^{3}$ http://www.atnf.csiro.au/research/pulsar/ppta/.

${ }^{4}$ http://www.leap.eu.org.

5 http://www.ipta4gw.org.

${ }^{6} \mathrm{www}$.lisamission.org.

${ }^{7} \mathrm{https}: / / \mathrm{www}$.elisascience.org.

${ }^{8}$ http://www.et-gw.eu.
}

distribution of sources are homogeneous and isotropic, see e.g., Refs. [23,54]. These assumptions can be relaxed in order to take into account that astrophysical sources are located in cosmic structures that indeed have a distribution that can be computed in a given cosmological model. Therefore the energy flux from all astrophysical sources (resolved and unresolved) is not constant across the sky and depends on the direction of observation. In [55] an analytic framework is presented to describe and compute the anisotropies in the observed energy density of the AGWB, taking into account the presence of inhomogeneities in the matter distribution and in the geometry of the observed universe. In [56] an alternative (more geometrical) derivation of the result of [55] is presented, and first numerical predictions for the amplitude of anisotropies for the contribution of the background coming from black hole mergers can be found in [57]. For an astrophysical background, the origin of anisotropies is two fold: first, sources are not isotropically distributed and second, a GW signal, once emitted, is deflected by structures. For a background of cosmological origin, lensing by large scale structures is the main source of anisotropy and actually, CGWB anisotropies are a tracer of $\mathrm{CMB}$ temperature anisotropies (see e.g., [58] for the case of a CGWB from phase transition). First constraints on the anisotropy have been obtained by PTA $[59,60]$, and from the first observing run of advanced LIGO [61].

In the framework developed in Refs. [55-57] the propagation of $\mathrm{GW}$ from the source to the observer is computed in the geometric optics approximation. This is also traditionally done for photons, e.g., to compute CMB temperature anisotropies or fluctuations of the galaxy distribution see e.g., [62]. The geometric optics approximation is justified as long as the wavelength of gravitons (or photons) is much smaller than the length scale given by the Kretschmann scalar of the metric describing the region of spacetime where the graviton (photon) propagates. In particular, for both gravitons and photons, this approximation is well motivated on cosmological distances, where spacetime is well described by a Friedmann-Lemaitre metric with scalar perturbations. In the geometric optics approximation, the polarization tensor is parallel transported along a geodesic: an initially unpolarized background stays unpolarized when it propagates in an inhomogeneous medium. In other words, geometric optics can not describe the generation of polarization. However, in the vicinity of a compact object, wavelike effects are present and sizable and the geometric optics approximation does not capture interesting effects like diffraction and the generation of polarization. In this regime a wavelike description of GW propagation is necessary. We emphasize that this result holds for $\mathrm{GW}$ where the wavelength of gravitons is typically much larger than the one of CMB or infrared photons. Therefore, when studying the propagation in a highly inhomogeneous medium, the analogy 
between the behavior of electromagnetic and gravitational radiation may break down.

In this paper we study the generation of polarization of an (initially unpolarized) GW background by diffusion through massive structures. We provide a framework to describe the generation of polarization and we derive an order of magnitude estimate of the polarization created by the interaction of GWs with matter, in different frequency bands. Our treatment bears several analogies with the creation of polarization by Thomson scattering of the CMB. The role played by electrons in the Thomson scattering is played here by compact and extended astrophysical objects (massive structures). In particular, as for the $\mathrm{CMB}$, it is the combined effect of anisotropies in the energy density of the background and of the polarizationdependence of the cross section effectively describing the process of scattering, that are responsible for the generation of polarization. The amplitude of the polarization generated depends on several factors: on the abundance of scattering centers (number density of massive structures), on the relative amplitude of anisotropies of the radiation impinging on a scattering center, on the wavelength of the GW and on the size of the integrated cross section describing the scattering off a given type of massive structure. This latter effect, in turn, depends on the geometrical properties of the astrophysical target (radius and mass) and on the wavelength. Interestingly, as we will explain in detail, by measuring the polarization of a given component of the background at different frequencies, it may be possible to set constraints on the abundance of some exotic and unresolvable sources in the Universe.

This work is structured as follows. In Sec. II we illustrate the general idea underlying our framework. In particular, in Secs. II A and II B we explain that beyond the geometric optics approximation, the interactions of GW with structures can be described as a diffusion process characterized by an polarization-dependent effective cross section. In Sec. II C we introduce the visibility function for a multi scattering process. In the remaining part of Sec. II we introduce the main ingredients needed to fully characterize the generation of polarization: in Sec. II D we define Stokes parameters for a GW background, in IIE we study the angular dependence of a (single) scattering event of a GW off an massive structure and in II F we compute the Stokes parameters after the scattering. Finally in II G we put all these ingredients together to derive an expression for the polarization tensor. In Sec. III we provide analytical approximations for the polarization tensor of a GW background (both astrophysical and cosmological) as a sum of contributions of scattering off different types of massive structures. In Sec. IV we present order of magnitude estimates of the amount of polarization generated by diffusion for cosmological and astrophysical backgrounds at different frequencies. Finally, in Sec. V we discuss our results and future perspectives.

\section{GENERAL FRAMEWORK}

As for the $\mathrm{CMB}$, the generation of polarization of a $\mathrm{GW}$ background occurs due to the combined effect of:

(i) the presence of anisotropies in the energy density of the background;

(ii) the dependence of the effective scattering cross section of GWs by massive structures on incoming direction and polarization.

In the following we explain in which regime the geometric optics approximation to describe GW propagation breaks down. This happens in the vicinity of a mass distribution in the form of compact (black hole) or extended objects, we denote them "massive structures." We then explain how to treat diffraction effects as an effective scattering process. When studying the angular dependence of the scattering process and computing the Stokes parameters after a scattering, Secs. II E and II F respectively, we make use of an approach similar to the standard description of CMB polarization in terms of Stokes parameters. In particular, we follow the pedagogical derivations presented in the textbook [63].

\section{A. Wavelike effects in GW propagation}

We write the metric describing the geometry of the spacetime as

$$
g_{\mu \nu}=\bar{g}_{\mu \nu}+h_{\mu \nu},
$$

where with an overbar we denote the background metric and $h_{\mu \nu}$ is a rapidly varying small perturbation on the top of it. Within linear perturbation theory, $h_{\mu \nu}$ satisfies the equation

$$
\square h_{\mu \nu}+2 \bar{R}_{\mu \alpha \nu \beta} h^{\alpha \beta}=0,
$$

where $\bar{R}_{\mu \alpha \nu \beta}$ is the Riemann tensor of the background metric and $\bar{\square}$ is the d'Alembertian of the background metric.

The geometric optics approximation consists in writing

$$
h_{\mu \nu}(x)=\left(A_{\mu \nu}(x)+\epsilon B_{\mu \nu}(x)+\ldots\right) e^{i \theta(x) / \epsilon},
$$

inserting it in (2.2) and keeping leading order terms in $\epsilon$. It is easy to verify that, in this approximation, the second term in Eq. (2.2) is systematically discarded. It follows that geometric optics is valid in the regime

$$
\frac{1}{\lambda^{2}} \gg \sqrt{\bar{R}_{\mu \alpha \nu \beta} \bar{R}^{\mu \alpha \nu \beta}} \equiv \sqrt{K},
$$

where $\lambda \sim\left(\partial_{0} \theta\right)^{-1}$ denotes a typical wavelength of the GW and the quantity on the right-hand side of this equation is the square root of the Kretschmann scalar of the metric $\bar{g}_{\mu \nu}$.

The vicinity of a compact object can be approximately described by a Schwarzschild metric with Kretschmann scalar $K(r)=12 r_{s}^{2} / r^{6}$, where $r_{s}=2 G M$ is the Schwarzschild radius of the mass $M$. The condition (2.4) defines a region around the object within which 
wavelike effects are present, given by $r_{s} \leq r \leq R_{\lambda}$ where $R_{\lambda}$ is defined by $K\left(R_{\lambda}\right)=\lambda^{-4}$, a length scale which depends on the mass of the object and on the wavelength of the GW under consideration. For a fixed mass, the size of this region obviously increases with increasing wavelength. Analogous conclusions hold for the case of an extended object: in this case $R_{\lambda}$ depends on both the mass and the radius of the object through a combination of these two quantities (compactness). In Sec. IVA, we will explicitly work out the wave-effect region for different types of astrophysical objects and for the wavelength range of current and planned GW experiments have access to.

Corrections of the geometric optics limit to the amplitude have also been considered in Ref. [64]. There, however the term $2 \bar{R}_{\mu \alpha \nu \beta} h^{\alpha \beta}$ has been neglected. Here we are interested in the polarization and we cannot neglect this term which affects the polarisation as we shall see.

\section{B. Effective treatment of scattering}

Let us consider a GW impinging on a black hole (extended object) of mass $M$ (and radius $R$ ), with impact parameter $b>r_{s}(b>R)$. The literature on gravitational scattering of massless waves of various spin is broad and stretches back over forty years; see the monograph [65] for an extensive treatment of the subject. Over the years, several authors using different methods (see Refs. [66-74]) have shown that the differential cross section (summed over polarizations) describing wave-scattering depends on the spin $s$ of the scattered field as

$$
\frac{1}{8(M G)^{2}} \frac{d \sigma}{d \Omega}= \begin{cases}\frac{1}{\sin ^{4} \theta / 2}, & s=0, \\ \frac{\cos ^{2} \theta / 2}{\sin ^{4} \theta / 2}, & s=1 / 2, \\ \frac{\cos ^{4} \theta / 2}{\sin ^{4} \theta / 2}, & s=1, \\ \frac{\cos ^{8} \theta / 2+\sin ^{8} \theta / 2}{\sin ^{4} \theta / 2}, & s=2,\end{cases}
$$

where $d \Omega \equiv d \cos \theta d \phi$ and $\theta$ is the scattering angle. The cross section for $s=2$ is the result of at least four separate studies. The first derivation was carried out in [67] applying perturbation theory to the linearized gravitational equations. The author of Ref. [68] finds the same result via a Green's function approach while in Ref. [70] Feynman diagram techniques are employed. Finally the author of Ref. [73] finds again the same result using partial wave methods and improves on previous work [66] which uses the same techniques. ${ }^{9}$

\footnotetext{
${ }^{9} \mathrm{We}$ emphasize that the gravitational result is somewhat anomalous since it does not follow the same general rule $d \sigma / d \Omega=M^{2} \cos ^{4 s} \theta / 2 / \sin ^{4} \theta / 2$ as other fields. As explained in [73], the origin of the extra term $\sin ^{4} \theta / 2$ is a direct consequence of the nonconservation of helicity in gravitational-wave scattering. Helicity is not conserved because axial and polar waves are scattered in a different way.
}

We observe that for arbitrary spin, the cross section diverges in the forward direction, i.e.,

$$
\frac{d \sigma}{d \Omega} \propto \frac{1}{\theta^{4}} \quad \text { for } \theta \rightarrow 0
$$

This divergence is due to the long-range nature of gravitational interactions and is present in every scattering process of a charged massless wave (or particle) in the Coulomb-like potential generated by a charged object. ${ }^{10}$ This divergence is physical and it is due to the fact that the Coulomb potential, that is used to model the gravitational potential of a massive object in a galaxy, is long-ranged. Nevertheless a natural cut-off scale is present in the problem under study. The far-field relation between deflection angle and impact parameter is given by

$$
\theta \approx \frac{2 r_{s}}{b}
$$

As we will see in detail in Sec. IV A, for both compact and extended objects wavelike effects occur in a region of space around the object of radius $r \leq R_{\lambda}$ where the parameter $R_{\lambda}$ depends on both the GW wavelength under consideration and the geometrical properties of the object. This sets an upper bound on the impact parameter $b_{\max }=R_{\lambda}$ and correspondingly a lower bound on the deflection angle

$$
\theta_{\min }=\frac{2 r_{s}}{b_{\max }}
$$

The results (2.5) are found assuming an unpolarized incoming flux and summing over the final polarization states. The polarization-dependent differential cross section for gravitational wave scattering is given by, see e.g., $[72,74]$

$$
\frac{d \sigma^{P}}{d \Omega}=(M G)^{2} \frac{1}{\sin ^{4} \theta / 2}\left|e_{i j}^{\prime}\left(\mathbf{n}^{\prime}\right) e^{* i j}(\mathbf{n})\right|^{2},
$$

where $\mathbf{n}^{\prime}$ and $\mathbf{n}$ are the directions of the incoming and outgoing gravitons, respectively and likewise for the polarizations, $e_{i j}^{\prime}$ and $e_{i j}$. The angle $\theta$ is the scattering angle, i.e., $\mathbf{n} \cdot \mathbf{n}^{\prime}=\cos \theta$. Using the results of Appendix A, it is easy to verify that the sum over polarizations of Eq. (2.9) gives back Eq. (2.5) for $s=2$.

\section{Visibility function}

We recall that if a particle scatters with a cross section $\sigma$ off an ensemble of targets with number density $n$, its mean free path is $\ell=1 /(n \sigma)$. In our case, gravitons scatter off

\footnotetext{
${ }^{10}$ The Rutherford cross section can be considered as the electromagnetic counterpart of (2.5) and presents the same type of divergence in the forward direction.
} 
astrophysical objects with physical number density $n_{\mathrm{ph}}$ and the relevant cross-section for scattering off different astrophysical objects is discussed in Sec. II B. One usually defines the optical depth due to scattering in the time interval $\left[\eta_{2}, \eta_{1}\right]$ by

$$
\tau\left(\eta_{1}, \eta_{2}\right)=\int_{\eta_{2}}^{\eta_{1}} d \eta n_{\mathrm{ph}}(\eta) \sigma(\eta) a(\eta)
$$

where $\eta$ is conformal time and $\eta_{1}>\eta_{2}$. The (unnormalized) probability that a graviton does not scatter off an astrophysical object in the conformal time interval $\left[\eta_{2}, \eta_{1}\right]$ is given by

$$
P\left(\eta_{1}, \eta_{2}\right)=e^{-\tau\left(\eta_{1}, \eta_{2}\right)}
$$

The probability density that a graviton observed at a time $\eta_{1}$ has undergone a scattering in the interval $\left[\eta_{2}, \eta_{2}+d \eta_{2}\right]$ is given by $P\left(\eta_{1}, \eta_{2}+d \eta_{2}\right)-P\left(\eta_{1}, \eta_{2}\right)$. We define the visibility function $\mathscr{V}\left(\eta_{1}, \eta_{2}\right)$ as follows:

$$
\mathscr{V}\left(\eta_{1}, \eta_{2}\right) d \eta_{2} \equiv P\left(\eta_{1}, \eta_{2}+d \eta_{2}\right)-P\left(\eta_{1}, \eta_{2}\right)
$$

It is easy to verify that

$$
\mathscr{V}\left(\eta_{1}, \eta_{2}\right)=\frac{d}{d \eta_{2}} e^{-\tau\left(\eta_{1}, \eta_{2}\right)}=-e^{-\tau\left(\eta_{1}, \eta_{2}\right)} \frac{d \tau\left(\eta_{1}, \eta_{2}\right)}{d \eta_{2}},
$$

with

$$
\frac{d \tau\left(\eta_{1}, \eta_{2}\right)}{d \eta_{2}}=-n_{\mathrm{ph}}\left(\eta_{2}\right) \sigma a\left(\eta_{2}\right)
$$

Hence

$$
\mathscr{V}\left(\eta_{1}, \eta_{2}\right)=e^{-\tau\left(\eta_{1}, \eta_{2}\right)} n_{\mathrm{ph}}\left(\eta_{2}\right) \sigma a\left(\eta_{2}\right)
$$

We are mostly interested in $\mathscr{V}(\eta)=\mathscr{V}\left(\eta_{0}, \eta\right)$ since we observe gravitons today. The quantity

$$
\int_{\eta}^{\eta_{0}} d \eta^{\prime} \mathscr{V}\left(\eta_{0}, \eta^{\prime}\right)=\left.e^{-\tau\left(\eta_{0}, \eta\right)}\right|_{\eta} ^{\eta_{0}}=1-e^{-\tau\left(\eta_{0}, \eta\right)},
$$

by construction is the probability that a graviton observed today has scattered in the time interval $\left[\eta, \eta_{0}\right]$.

A similar treatment is used for the $\mathrm{CMB}$, see e.g., Chapter 20 of [63] which we have followed here, by substituting $n_{\mathrm{ph}} \rightarrow n_{e}$ and $\sigma \rightarrow \sigma_{T}$, where $n_{e}$ is the density of electrons and $\sigma_{T}$ is the Thomson cross section. In the case of standard recombination, the visibility function is peaked around recombination. Indeed, before recombination, $\tau$ is very large and $\mathscr{V}$ is exponentially suppressed. Much later, $d \tau\left(\eta_{0}, \eta\right) / d \eta$ is small because the density of free electrons is small. The width of the maximum of the visibility function gives the thickness of the last scattering surface.

For a GW background the distribution of scattering centers is extended in redshift and thus the situation is different. In that case we expect the visibility function to be much broader and peaked around a redshift at which most of the astrophysical objects are expected to be located. This is similar to what happens during reionization for the CMB. Moreover, for the CMB, since $\tau\left(\eta_{0}, \eta_{0}\right)=0$ and at early times, say $\eta=0, \tau\left(\eta_{0}, 0\right) \rightarrow \infty$, the visibility function satisfies

$$
\int_{0}^{\eta_{0}} d \eta \mathscr{V}_{\mathrm{CMB}}\left(\eta_{0}, \eta\right)=\left.e^{-\tau\left(\eta_{0}, \eta\right)}\right|_{0} ^{\eta_{0}}=1
$$

Thus $\mathscr{V}_{\mathrm{CMB}}(\eta)$ is the normalized probability function that a photon observed today has scattered at conformal time $\eta$. This is as expected since the total probability that a CMB photon scatters before impinging on an observer equals to one. This property is not, however, true for a $\mathrm{GW}$ background.

\section{Stokes parameters for GW background}

In this section we introduce the Stokes parameters to describe the intensity and polarization of a GW background, see also [47,75-77]. For a single monochromatic plane wave propagating in direction $\mathbf{n}$, we have that

$$
\tilde{h}_{i j}(f, \mathbf{n})=\tilde{h}_{+}(f, \mathbf{n}) e_{i j}^{+}(\mathbf{n})+\tilde{h}_{\times}(f, \mathbf{n}) e_{i j}^{\times}(\mathbf{n}),
$$

where the expansion coefficients $h_{+, \times}$are complex-valued functions and $e_{i j}^{+, \times}$is the $(+, \times)$polarization basis (see Appendix A for detailed definitions). We can introduce a polarization tensor as

$$
P_{i j k l}=\tilde{\mathcal{P}}_{a b} e_{i j}^{a} e_{k l}^{b}, \quad \text { with } \quad \tilde{\mathcal{P}}_{a b}=\tilde{h}_{a}^{*} \tilde{h}_{b},
$$

where $a, b=(+, \times)$. The tensor $\tilde{\mathcal{P}}_{a b}$ is a Hermitian $2 \times 2$ matrix and therefore can be written as

$$
\begin{aligned}
\tilde{\mathcal{P}}_{a b}(\mathbf{n}, f)= & \frac{1}{2}\left[I(\mathbf{n}, f) \sigma_{a b}^{(0)}+U(\mathbf{n}, f) \sigma_{a b}^{(1)}\right. \\
& \left.+V(\mathbf{n}, f) \sigma_{a b}^{(2)}+Q(\mathbf{n}, f) \sigma_{a b}^{(3)}\right],
\end{aligned}
$$

where $\sigma^{(\alpha)}$ with $\alpha=1,2,3$ denote the Pauli matrices and $\sigma^{(0)}=1_{2}$ (i.e., the $2 \times 2$ identity matrix). The objects $I, U$, $Q, V$ are four real functions of the $\mathrm{GW}$ direction $\mathbf{n}$ and are the Stokes parameters. In terms of the polarization coefficients of the GW, the Stokes parameters are given by

$$
\begin{aligned}
I & =\left|\tilde{h}_{+}\right|^{2}+\left|\tilde{h}_{\times}\right|^{2}, \quad Q=\left|\tilde{h}_{+}\right|^{2}-\left|\tilde{h}_{\times}\right|^{2}, \\
U & =2 \operatorname{Re}\left(\tilde{h}_{+}^{*} \tilde{h}_{\times}\right), \quad V=2 \operatorname{Im}\left(\tilde{h}_{+}^{*} \tilde{h}_{\times}\right) .
\end{aligned}
$$

The Stokes parameter $I$ is simply the intensity of the GW, $Q$ is the difference between the intensity of radiation polarized 
along $e_{i j}^{+}$and $e_{i j}^{\times}$(and $U$ is the same in a frame rotated ${ }^{11}$ by $\pi / 8)$. The parameter $V$ describes a phase difference between $h_{+}$and $h_{\times}$which results in circular polarization. Using Eqs. (A4) and (A5), we can rewrite these parameters in terms of the left and right-handed polarization basis defined as

$$
\begin{aligned}
& e_{i j}^{R}(\mathbf{n})=\frac{1}{\sqrt{2}}\left(e_{i j}^{+}(\mathbf{n})+i e_{i j}^{\times}(\mathbf{n})\right), \\
& e_{i j}^{L}(\mathbf{n})=\frac{1}{\sqrt{2}}\left(e_{i j}^{+}(\mathbf{n})-i e_{i j}^{\times}(\mathbf{n})\right) .
\end{aligned}
$$

We obtain

$$
\begin{aligned}
I & =\left|\tilde{h}_{L}\right|^{2}+\left|\tilde{h}_{R}\right|^{2}, \quad Q=2 \operatorname{Re}\left(\tilde{h}_{R}^{*} \tilde{h}_{L}\right), \\
U & =2 \operatorname{Im}\left(\tilde{h}_{R}^{*} \tilde{h}_{L}\right), \quad V=\left|\tilde{h}_{R}\right|^{2}-\left|\tilde{h}_{L}\right|^{2} .
\end{aligned}
$$

It is useful to introduce the following tensor

$$
\mathcal{P}_{a b}(\mathbf{n}, f)=\left[\mathcal{U}(\mathbf{n}, f) \sigma_{a b}^{(1)}+\mathcal{V}(\mathbf{n}, f) \sigma_{a b}^{(2)}+\mathcal{Q}(\mathbf{n}, f) \sigma_{a b}^{(3)}\right],
$$

in terms of the normalized Stokes parameters $\mathcal{U}=U /(2 I)$, $\mathcal{Q}=Q /(2 I)$ and $\mathcal{V}=V /(2 I)$. We can compute the total amplitude of polarization as

$$
P(\mathbf{n}, f) \equiv \frac{1}{\sqrt{2}}\left(\mathcal{P}_{a b}(\mathbf{n}, f) \mathcal{P}^{b a}(\mathbf{n}, f)\right)^{1 / 2}=\sqrt{\mathcal{Q}^{2}+\mathcal{U}^{2}+\mathcal{V}^{2}},
$$

Under a rotation of an angle $\psi$ around $\mathbf{n}$, using the transformation properties of (A10) and (A11), we find (omitting the dependence on frequency)

$$
\begin{gathered}
\tilde{h}_{R}(\mathbf{n} ; \psi)=e^{i 2 \psi} \tilde{h}_{R}(\mathbf{n}), \\
\tilde{h}_{L}(\mathbf{n} ; \psi)=e^{-i 2 \psi} \tilde{h}_{L}(\mathbf{n}) .
\end{gathered}
$$

It follows that under rotation in the plane orthogonal to $\mathbf{n}$, the Stokes parameters transform as (omitting the dependence on frequency)

$$
\begin{gathered}
I(\mathbf{n} ; \psi)=I(\mathbf{n}), \\
V(\mathbf{n} ; \psi)=V(\mathbf{n}), \\
Q(\mathbf{n} ; \psi)+i U(\mathbf{n} ; \psi)=e^{-i 4 \psi}(Q(\mathbf{n})+i U(\mathbf{n})), \\
Q(\mathbf{n} ; \psi)-i U(\mathbf{n} ; \psi)=e^{i 4 \psi}(Q(\mathbf{n})-i U(\mathbf{n})) .
\end{gathered}
$$

\footnotetext{
${ }^{11}$ Note that for spin 1 particles, photons, $U$ describes the polarization rotated by $\pi / 4$ but for gravitons a rotation by $\pi / 4$ simply exchanges $h_{+}$and $h_{\times}$, hence $Q \mapsto-Q$.
}

From this, together with the fact that the Stokes parameters are real, we easily conclude that a pure $Q$ polarization turns into a pure $U$ polarization under a rotation by $\pm \pi / 8$ and vice versa. This proves footnote 11 . Furthermore, $I$ and $V$ transform as scalars on the sphere under rotations while $Q \pm i U$ are spin-4 objects and can be written as linear combinations of spin-4 spherical harmonics. In particular, one can write

$$
\left(\begin{array}{c}
Q(\mathbf{n} ; \psi) \\
U(\mathbf{n} ; \psi)
\end{array}\right)=R(4 \psi)\left(\begin{array}{c}
Q(\mathbf{n}) \\
U(\mathbf{n})
\end{array}\right)
$$

where $R(4 \psi)$ is a rotation matrix describing a rotation around the $\mathbf{n}$ axis.

\section{E. Angular dependence of the scattering process}

If we have a flux of unpolarized radiation coming from a given direction and impinging on a massive object, the dependence of the cross section on the polarization tensors generates an outgoing polarized radiation. As a first step we compute the net polarization generated when a radial flux of gravitons whose intensity has a given angular dependence $I\left(\theta^{\prime}, \phi^{\prime}\right)$ scatters off a massive object at the origin of our reference frame. We consider an incoming graviton whose propagation direction is

$$
\mathbf{n}^{\prime}=\left(\sin \theta^{\prime} \sin \phi^{\prime}, \sin \theta^{\prime} \cos \phi^{\prime}, \cos \theta^{\prime}\right),
$$

i.e., $\mathbf{n}^{\prime}$ is the unit radial vector with angles $\left(\theta^{\prime}, \phi^{\prime}\right)$. In the plane transverse to $\mathbf{n}^{\prime}$ we introduce two orthonormal vectors

$$
\begin{gathered}
\mathbf{u}^{\prime}=\left(\cos \theta^{\prime} \sin \phi^{\prime}, \cos \theta^{\prime} \cos \phi^{\prime},-\sin \theta^{\prime}\right), \\
\mathbf{v}^{\prime}=\left(\cos \phi^{\prime},-\sin \phi^{\prime}, 0\right) .
\end{gathered}
$$

Using these vectors and Eq. (A12) we construct the polarization basis $\left(e_{i j}^{\times}\left(\mathbf{n}^{\prime}\right), e_{i j}^{+}\left(\mathbf{n}^{\prime}\right)\right)$ for the incoming radiation. Let us choose a reference frame such that the direction of propagation of the outgoing radiation $\mathbf{n}$, is along the $z$ axis, i.e., $\mathbf{n}=\mathbf{e}_{z}$. Then we can chose $\mathbf{u}=\mathbf{e}_{x}$ and $\mathbf{v}=\mathbf{e}_{y}$ for the polarization basis $\left(e_{i j}^{\times}(\mathbf{n}), e_{i j}^{+}(\mathbf{n})\right)$ of the outgoing radiation, using Eq. (A12).

If the incoming radiation has $\times$ polarization then the probability that the outgoing radiation has $\times$ polarization is proportional to ${ }^{12}$

$$
\left|e_{i j}^{\times}\left(\mathbf{n}^{\prime}\right) e^{i j \times}(\mathbf{n})\right|^{2}=4 \cos ^{2} \theta^{\prime} \cos ^{2} 2 \phi^{\prime} .
$$

Analogously, if the incoming radiation has $\times$ polarization then the probability that the outgoing radiation has + polarization is proportional to

\footnotetext{
${ }^{12}$ We recall that the basis $(+, \times)$ is real.
} 


$$
\left|e_{i j}^{\times}\left(\mathbf{n}^{\prime}\right) e^{i j+}(\mathbf{n})\right|^{2}=4 \cos ^{2} \theta^{\prime} \sin ^{2} 2 \phi^{\prime} .
$$

If the incoming radiation has + polarization then the probability that the outgoing radiation has $x$ and + polarizations is proportional to, respectively

$$
\begin{aligned}
\left|e_{i j}^{+}\left(\mathbf{n}^{\prime}\right) e^{i j \times}(\mathbf{n})\right|^{2} & =\frac{1}{4}\left(3+\cos 2 \theta^{\prime}\right)^{2} \sin ^{2} 2 \phi^{\prime}, \\
\left|e_{i j}^{+}\left(\mathbf{n}^{\prime}\right) e^{i j+}(\mathbf{n})\right|^{2} & =\frac{1}{4}\left(3+\cos 2 \theta^{\prime}\right)^{2} \cos ^{2} 2 \phi^{\prime} .
\end{aligned}
$$

If the initial radiation is unpolarized, there is an equal probability that the incoming graviton has $\times$ or + polarization. It follows that the probability that a graviton is scattered out with $\times$ polarization is given by

$\mathcal{E}_{\times}^{2} \equiv C\left(\mathbf{n}, \mathbf{n}^{\prime}\right)\left[\left|e_{i j}^{\times}\left(\mathbf{n}^{\prime}\right) e^{i j \times}(\mathbf{n})\right|^{2}+\left|e_{i j}^{+}\left(\mathbf{n}^{\prime}\right) e^{i j \times}(\mathbf{n})\right|^{2}\right]$,

while the probability that the incoming radiation is scattered out with + polarization is given by

$\mathcal{E}_{+}^{2} \equiv C\left(\mathbf{n}, \mathbf{n}^{\prime}\right)\left[\left|e_{i j}^{\times}\left(\mathbf{n}^{\prime}\right) e^{i j+}(\mathbf{n})\right|^{2}+\left|e_{i j}^{+}\left(\mathbf{n}^{\prime}\right) e^{i j+}(\mathbf{n})\right|^{2}\right]$,

where $C\left(\mathbf{n}, \mathbf{n}^{\prime}\right)$ is the prefactor multiplying the polarization-dependent part of the cross section, Eq. (2.9), and is given by

$$
C\left(\mathbf{n}, \mathbf{n}^{\prime}\right)=C \sin ^{-4} \frac{\theta^{\prime}}{2}
$$

where $C$ is a constant which does not depend on angles and which disappears in the final computation of the polarization tensor. Explicitly, one finds

$$
\begin{aligned}
& \mathcal{E}_{\times}^{2}=C \frac{1}{\sin ^{4} \theta^{\prime} / 2}\left[4\left(\cos ^{8} \frac{\theta^{\prime}}{2}+\sin ^{8} \frac{\theta^{\prime}}{2}\right)-\frac{1}{2} \cos 4 \phi^{\prime} \sin ^{4} \theta^{\prime}\right], \\
& \mathcal{E}_{+}^{2}=C \frac{1}{\sin ^{4} \theta^{\prime} / 2}\left[4\left(\cos ^{8} \frac{\theta^{\prime}}{2}+\sin ^{8} \frac{\theta^{\prime}}{2}\right)+\frac{1}{2} \cos 4 \phi^{\prime} \sin ^{4} \theta^{\prime}\right] .
\end{aligned}
$$

\section{F. Stokes parameters after scattering}

The Stokes parameter $I$ of the radiation scattered in the $\mathbf{e}_{z}$ direction is obtained by integrating $\mathcal{E}_{\times}^{2}+\mathcal{E}_{+}^{2}$ over all directions of the incoming radiation, weighted with the intensity of the incoming radiation $I\left(\theta^{\prime}, \phi^{\prime}\right)$ :

$$
I=C \int d \Omega^{\prime} I\left(\theta^{\prime}, \phi^{\prime}\right) \frac{8}{\sin ^{4} \theta^{\prime} / 2}\left(\cos ^{8} \frac{\theta^{\prime}}{2}+\sin ^{8} \frac{\theta^{\prime}}{2}\right) .
$$

The Stokes parameter $Q$ is obtained by integrating $\mathcal{E}_{+}^{2}-\mathcal{E}_{\times}^{2}$ over directions, again with $I\left(\theta^{\prime}, \phi^{\prime}\right)$ as a weight:

$$
Q=C \int d \Omega^{\prime} I\left(\theta^{\prime}, \phi^{\prime}\right) \frac{1}{\sin ^{4} \theta^{\prime} / 2} \cos 4 \phi^{\prime} \sin ^{4} \theta^{\prime} .
$$

As explained in Sec. II D, the Stokes parameter $U$ can be obtained from $Q$ with a rotation around the $\mathbf{n}$ axis of $\pi / 8^{13}$

$U=-C \int d \Omega^{\prime} I\left(\theta^{\prime}, \phi^{\prime}\right) \frac{1}{\sin ^{4} \theta^{\prime} / 2} \sin 4 \phi^{\prime} \sin ^{4} \theta^{\prime}$.

The Stokes parameter $V$ is defined as the difference between the intensity polarized $R$ and $L$, see Sec. II D. We can build a basis $\left(e_{i j}^{L}\left(\mathbf{n}^{\prime}\right), e_{i j}^{R}\left(\mathbf{n}^{\prime}\right)\right)$ for the incoming radiation and a basis $\left(e_{i j}^{L}(\mathbf{n}), e_{i j}^{R}(\mathbf{n})\right)$ for the outgoing radiation, starting from the basis $(+, \times)$ and using Eq. (2.22). Explicitly

$$
\begin{aligned}
& e_{i j}^{R}(\mathbf{n})=\frac{1}{\sqrt{2}}\left(e_{i j}^{+}(\mathbf{n})+i e_{i j}^{\times}(\mathbf{n})\right), \\
& e_{i j}^{L}(\mathbf{n})=\frac{1}{\sqrt{2}}\left(e_{i j}^{+}(\mathbf{n})-i e_{i j}^{\times}(\mathbf{n})\right),
\end{aligned}
$$

and analogously for the incoming radiation by replacing $\mathbf{n} \rightarrow \mathbf{n}^{\prime}$. The probability that an initially unpolarized radiation is polarized $R(L)$ after the scattering is given by

$$
\begin{aligned}
& \mathcal{E}_{R}^{2} \equiv C\left[\left|e_{i j}^{R}\left(\mathbf{n}^{\prime}\right) e^{i j R}(\mathbf{n})\right|^{2}+\left|e_{i j}^{L}\left(\mathbf{n}^{\prime}\right) e^{i j R}(\mathbf{n})\right|^{2}\right], \\
& \mathcal{E}_{L}^{2} \equiv C\left[\left|e_{i j}^{R}\left(\mathbf{n}^{\prime}\right) e^{i j L}(\mathbf{n})\right|^{2}+\left|e_{i j}^{L}\left(\mathbf{n}^{\prime}\right) e^{i j L}(\mathbf{n})\right|^{2}\right],
\end{aligned}
$$

respectively. It is easy to verify that

$$
\mathcal{E}_{R}^{2}=\mathcal{E}_{L}^{2}
$$

It follows that

$$
V=C \int d \Omega^{\prime} I\left(\theta^{\prime}, \phi^{\prime}\right) \frac{1}{\sin ^{4} \theta^{\prime} / 2}\left(\mathcal{E}_{R}^{2}-\mathcal{E}_{L}^{2}\right)=0,
$$

the scattering of GW radiation off a massive object does not generate circular polarization, in full analogy with the Thomson scattering for electromagnetic radiation.

Summarizing, we found the following Stokes parameters for the $\mathrm{GW}$ radiation along $\mathbf{n}=\mathbf{e}_{z}$, after the scattering of unpolarized radiation off a massive object ${ }^{14}$

\footnotetext{
${ }^{13}$ More precisely, $U$ can be obtained from $Q$ by projecting on a polarization basis built from $\mathbf{u}$ and $\mathbf{v}$ vectors rotated of $\pi / 8$ in the plane perpendicular to $\mathbf{n}$.

${ }^{14}$ Note that the integral over the scattering angle $\theta^{\prime}$ has a lower bound $\theta_{\min }$ proportional to the mass of the scatterer and to the frequency of the GW scattering off it, see Secs. II B and IV B.
} 


$$
\begin{gathered}
I=C \int d \Omega^{\prime} I\left(\theta^{\prime}, \phi^{\prime}\right) \frac{8}{\sin ^{4} \theta^{\prime} / 2}\left[\cos ^{8} \frac{\theta^{\prime}}{2}+\sin ^{8} \frac{\theta^{\prime}}{2}\right], \\
Q=C \int d \Omega^{\prime} I\left(\theta^{\prime}, \phi^{\prime}\right) \frac{1}{\sin ^{4} \theta^{\prime} / 2}\left[\cos 4 \phi^{\prime} \sin ^{4} \theta^{\prime}\right], \\
U=C \int d \Omega^{\prime} I\left(\theta^{\prime}, \phi^{\prime}\right) \frac{1}{\sin ^{4} \theta^{\prime} / 2}\left[-\sin 4 \phi^{\prime} \sin ^{4} \theta^{\prime}\right], \\
V=0 .
\end{gathered}
$$

The angular factors in square parenthesis in Eqs. (2.56) and (2.57) can be expanded in a basis of spherical harmonics with $m= \pm 4$ and $\ell \geq 4$. It follows that an isotropic incoming flux of radiation does not generate any net polarization.

Up to now we have chosen the coordinate system such that the direction of propagation of the outgoing radiation was along the $z$-axis. We now rewrite Eqs. (2.55)-(2.58) in a coordinate independent (rotationally invariant) way. First we make use of standard trigonometric identities to rewrite (2.55)-(2.57) as a functions of $\cos \theta^{\prime}, \sin \theta^{\prime}$ only. Then we introduce two orthonormal vectors in the plane perpendicular to $\mathbf{n}, \mathbf{u}(\mathbf{n})$ and $\mathbf{v}(\mathbf{n})$. For the choice $\mathbf{n}=\mathbf{e}_{z}$ they reduce to $\mathbf{u}\left(\mathbf{e}_{z}\right)=\mathbf{e}_{x}$ and $\mathbf{v}\left(\mathbf{e}_{z}\right)=\mathbf{e}_{y}$. With these definitions, we have

$$
\begin{gathered}
\cos \theta^{\prime}=\mathbf{n} \cdot \mathbf{n}^{\prime}, \\
\sin \phi^{\prime}=\frac{\mathbf{n}^{\prime} \cdot \mathbf{u}(\mathbf{n})}{\sqrt{1-\left(\mathbf{n} \cdot \mathbf{n}^{\prime}\right)^{2}}} \\
\cos \phi^{\prime}=\frac{\mathbf{n}^{\prime} \cdot \mathbf{v}(\mathbf{n})}{\sqrt{1-\left(\mathbf{n} \cdot \mathbf{n}^{\prime}\right)^{2}}} .
\end{gathered}
$$

After standard simplifications, we find

$$
\begin{aligned}
I(\mathbf{n})= & C \int d^{2} \mathbf{n}^{\prime} I\left(\mathbf{n}^{\prime}\right) \frac{2}{\left[1-\mathbf{n} \cdot \mathbf{n}^{\prime}\right]^{2}} \\
& \times\left[\left(1+\mathbf{n} \cdot \mathbf{n}^{\prime}\right)^{4}+\left(1-\mathbf{n} \cdot \mathbf{n}^{\prime}\right)^{4}\right], \\
Q(\mathbf{n})= & C \int d^{2} \mathbf{n}^{\prime} I\left(\mathbf{n}^{\prime}\right) \frac{4}{\left[1-\mathbf{n} \cdot \mathbf{n}^{\prime}\right]^{2}} \\
& \times\left[\left(\mathbf{n}^{\prime} \cdot \mathbf{v}_{\mathbf{n}}\right)^{4}+\left(\mathbf{n}^{\prime} \cdot \mathbf{u}_{\mathbf{n}}\right)^{4}-6\left(\mathbf{n}^{\prime} \cdot \mathbf{v}_{\mathbf{n}}\right)^{2}\left(\mathbf{n}^{\prime} \cdot \mathbf{u}_{\mathbf{n}}\right)^{2}\right],
\end{aligned}
$$

$$
\begin{aligned}
& U(\mathbf{n})= C \int d^{2} \mathbf{n}^{\prime} I\left(\mathbf{n}^{\prime}\right) \frac{16}{\left[1-\mathbf{n} \cdot \mathbf{n}^{\prime}\right]^{2}} \\
& \times\left[\left(\mathbf{n}^{\prime} \cdot \mathbf{u}_{\mathbf{n}}\right)^{3}\left(\mathbf{n}^{\prime} \cdot \mathbf{v}_{\mathbf{n}}\right)-\left(\mathbf{n}^{\prime} \cdot \mathbf{v}_{\mathbf{n}}\right)^{3}\left(\mathbf{n}^{\prime} \cdot \mathbf{u}_{\mathbf{n}}\right)\right], \\
& V(\mathbf{n})=0,
\end{aligned}
$$

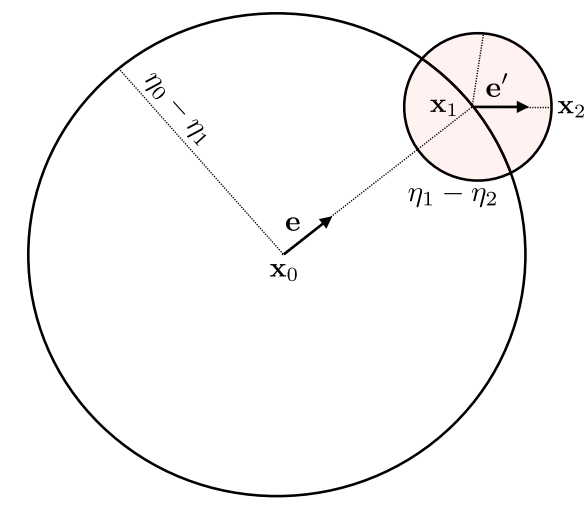

FIG. 1. Schematic representation of the scattering processes under study. The observer located in $\mathbf{x}_{0}$ is looking at the sky in the direction $\mathbf{e}$ and receives GW which have scattered out of an astrophysical object located in $\mathbf{x}_{1}=\mathbf{x}_{0}+\mathbf{e}\left(\eta_{0}-\eta_{1}\right)$, coming from $\mathbf{x}_{2}=\mathbf{x}_{1}+\mathbf{e}^{\prime}\left(\eta_{2}-\eta_{1}\right)$. Readapted from [63].

where we have simplified notation to $\mathbf{u}_{\mathbf{n}}=\mathbf{u}(\mathbf{n})$ and $\mathbf{v}_{\mathbf{n}}=\mathbf{v}(\mathbf{n})$. Equations (2.62)-(2.65) determine the Stokes parameter of the GW radiation scattered by a black hole into direction $\mathbf{n}$.

Consider the scattering geometry in Fig. 1 where we neglect lensing effects and the observer receives the outgoing radiation in the direction $\mathbf{e}=-\mathbf{n}$. The Stokes parameter which can be measured in the direction of observation e are therefore given by

$$
\begin{gathered}
I(\mathbf{e})=C \int d^{2} \mathbf{e}^{\prime} I\left(\mathbf{e}^{\prime}\right) \frac{2}{\left[1-\mathbf{e} \cdot \mathbf{e}^{\prime}\right]^{2}} \\
\times\left[\left(1+\mathbf{e} \cdot \mathbf{e}^{\prime}\right)^{4}+\left(1-\mathbf{e} \cdot \mathbf{e}^{\prime}\right)^{4}\right] \\
Q(\mathbf{e})=C \int d^{2} \mathbf{e}^{\prime} I\left(\mathbf{e}^{\prime}\right) \frac{4}{\left[1-\mathbf{e} \cdot \mathbf{e}^{\prime}\right]^{2}} \\
\times\left[\left(\mathbf{e}^{\prime} \cdot \mathbf{v}_{\mathbf{e}}\right)^{4}+\left(\mathbf{e}^{\prime} \cdot \mathbf{u}_{\mathbf{e}}\right)^{4}-6\left(\mathbf{e}^{\prime} \cdot \mathbf{v}_{\mathbf{e}}\right)^{2}\left(\mathbf{e}^{\prime} \cdot \mathbf{u}_{\mathbf{e}}\right)^{2}\right] \\
U(\mathbf{e})= \\
C \int d^{2} \mathbf{e}^{\prime} I\left(\mathbf{e}^{\prime}\right) \frac{16}{\left[1-\mathbf{e} \cdot \mathbf{e}^{\prime}\right]^{2}} \\
\times\left[\left(\mathbf{e}^{\prime} \cdot \mathbf{u}_{\mathbf{e}}\right)^{3}\left(\mathbf{e}^{\prime} \cdot \mathbf{v}_{\mathbf{e}}\right)-\left(\mathbf{e}^{\prime} \cdot \mathbf{v}_{\mathbf{e}}\right)^{3}\left(\mathbf{e}^{\prime} \cdot \mathbf{u}_{\mathbf{e}}\right)\right] \\
V(\mathbf{e})=0
\end{gathered}
$$

where $\mathbf{e}^{\prime}=-\mathbf{n}^{\prime}$ and $\mathbf{v}_{\mathbf{e}}=\mathbf{v}(\mathbf{e})=\mathbf{v}(-\mathbf{n})$ and $\mathbf{u}_{\mathbf{e}}=\mathbf{u}(\mathbf{e})=$ $\mathbf{u}(-\mathbf{n})$ are orthonormal vectors in the plane perpendicular to $\mathbf{e}$ such that $\mathbf{v}\left(\mathbf{e}=\mathbf{e}_{z}\right)=-\mathbf{e}_{y}$ and $\mathbf{u}\left(\mathbf{e}=\mathbf{e}_{z}\right)=-\mathbf{e}_{x}$.

The Stokes parameters still depend on the choice of the directions $\mathbf{v}_{\mathbf{e}}$ and $\mathbf{u}_{\mathbf{e}}$. This can be avoided by expanding the polarisation into $E$-mode (gradient type) and $B$-mode (curl type) components analogous to the total angular momentum decomposition for the CMB (see e.g., [78]), but we refrain from this further formal development here. 


\section{G. Polarization tensor}

We split the intensity into an homogeneous and isotropic contribution and an inhomogeneous and anisotropic one. For gravitational radiation of (observed) frequency $f$ impinging on a scattering center at $\mathbf{x}$ in the direction $\mathbf{e}$ and at time $\eta$, we write

$$
I(\eta, \mathbf{x}, \mathbf{e}, f)=\bar{I}(\eta, f)+\delta I(\eta, \mathbf{x}, \mathbf{e}, f) .
$$

At linear order in $\delta I$, the polarization tensor (2.25) is given by

$$
\begin{aligned}
& \mathcal{P}_{a b}\left(\eta_{0}, \mathbf{x}_{0}, \mathbf{e}, f\right) \\
& \quad=\frac{\int_{0}^{\eta_{0}} d \eta_{1} \mathscr{V}\left(\eta_{0}, \eta_{1}\right) \int d^{2} \mathbf{e}^{\prime} \delta I\left(\eta_{1}, \mathbf{x}_{1}, \mathbf{e}^{\prime}, f_{1}\right) \mathcal{S}_{a b}\left(\mathbf{e}, \mathbf{e}^{\prime}\right)}{2 \bar{I}\left(\eta_{0}, \mathbf{x}_{0}, f\right)},
\end{aligned}
$$

where $\mathbf{x}_{1}=\mathbf{x}_{0}+\left(\eta_{0}-\eta_{1}\right) \mathbf{e}$ is the position of the scattering center, see Fig. $1, f_{1}=\left(1+z\left(\eta_{1}\right)\right) f$ and we have defined the following quantity which depends only on angles

$$
\begin{aligned}
& S_{a b}\left(\mathbf{e}, \mathbf{e}^{\prime}\right) \\
& =\frac{4}{\left[1-\mathbf{e} \cdot \mathbf{e}^{\prime}\right]^{2}}\left\{4\left[\left(\mathbf{e}^{\prime} \cdot \mathbf{u}_{\mathbf{e}}\right)^{3}\left(\mathbf{e}^{\prime} \cdot \mathbf{v}_{\mathbf{e}}\right)-\left(\mathbf{e}^{\prime} \cdot \mathbf{v}_{\mathbf{e}}\right)^{3}\left(\mathbf{e}^{\prime} \cdot \mathbf{u}_{\mathbf{e}}\right)\right] \sigma_{a b}^{(1)}\right. \\
& \left.\quad+\left[\left(\mathbf{e}^{\prime} \cdot \mathbf{v}_{\mathbf{e}}\right)^{4}+\left(\mathbf{e}^{\prime} \cdot \mathbf{u}_{\mathbf{e}}\right)^{4}-6\left(\mathbf{e}^{\prime} \cdot \mathbf{v}_{\mathbf{e}}\right)^{2}\left(\mathbf{e}^{\prime} \cdot \mathbf{u}_{\mathbf{e}}\right)^{2}\right] \sigma_{a b}^{(3)}\right\} .
\end{aligned}
$$

In Eq. (2.71), $I\left(\eta_{1}, \mathbf{x}_{1}, \mathbf{e}^{\prime}, f_{1}\right)$ is the intensity of the radiation incident on the scattering center in $\left(\eta_{1}, \mathbf{x}_{1}\right)$, from the direction $\mathbf{e}^{\prime}$ and at frequency $f_{1} . \bar{I}\left(\eta_{0}, \mathbf{x}_{0}, f\right)$ is the intensity at the observer (averaged over directions). As we will explain in the next section, while polarization is generated only by wavelike effects, the intensity does not vanish in the geometric optics approximation either (i.e., even if diffraction is discarded).

\section{ANALYTIC EXPRESSIONS FOR POLARIZATION}

We want to derive an expression for the polarization tensor $\mathcal{P}_{a b}$, Eq. (2.71), for a primordial (cosmological) background and for an astrophysical background, both in a cosmological setting (i.e., a Friedmann universe with structures). The ingredients needed are

(1) the visibility function for different types of scattering, introduced in Sec. II C;

(2) the intensity of the incoming radiation.

In Sec. III A we write the intensity of the incoming radiation in terms of the energy density of the background. The energy density at a point $\left(\eta_{1}, \mathbf{x}_{1}\right)$ of the space time, and seen in a direction $\mathbf{e}_{1}$ by a comoving observer in this position, can be computed by using the Boltzmann approach detailed in Appendix B.
Our approach is as follows. The intensity of the background is computed in the geometric optics approximation, neglecting diffusion effects. In the Boltzmann equation approach, this corresponds to neglecting the collision term and solving the Liouville equation with an emissivity part only. We then use this result to compute the polarization, generated by diffusion. Polarization is a purely beyond-geometric optics effect. On the other hand wavelike effects represent a second order correction to the intensity and we neglect them in the present treatment. ${ }^{15}$

We consider the standard cosmological framework in which the universe is modeled by a Friedmann-LemaîtreRobertson-Walker (FLRW) universe with Euclidean spatial sections and with scalar perturbations. In Newtonian gauge, the metric $g_{\mu \nu}$ is given by

$\mathrm{d} s^{2}=a(\eta)^{2}\left[-(1+2 \psi) \mathrm{d} \eta^{2}+(1-2 \phi) \delta_{i j} \mathrm{~d} x^{i} \mathrm{~d} x^{j}\right]$,

where the metric of the constant time hypersurfaces is

$$
\delta_{i j} \mathrm{~d} x^{i} \mathrm{~d} x^{j}=\mathrm{d} \chi^{2}+\chi^{2}\left(\mathrm{~d} \theta^{2}+\sin ^{2} \theta \mathrm{d} \phi^{2}\right)
$$

in terms of the comoving radial distance $\chi$. The two Bardeen potentials are decomposed as

$$
\psi=\Psi+\Pi, \quad \phi=\Psi-\Pi .
$$

In the standard $\Lambda \mathrm{CDM}$ model, the matter content at late time is dominated by cold dark matter (CDM), described by a pressureless fluid, and by the cosmological constant. It follows that the Bardeen potentials, $\phi$ and $\psi$, are equal, so that $\psi=\phi=\Psi$ and $\Pi=0$. We assume that the galaxies are all comoving with the cosmic flow. ${ }^{16}$ To first order in perturbations, the four velocity of the cosmic fluid is given by

$$
u^{\mu} \equiv \frac{1}{a}\left(1-\psi, v^{i}\right) \equiv \bar{u}^{\mu}+\delta u^{\mu}
$$

where $v^{i}$ is the peculiar velocity field. From the matter conservation equation, the galaxy peculiar velocity can be related to the gravitational potential through the Euler equation.

\footnotetext{
${ }^{15}$ Writing a set of Boltzmann equations for intensity and polarization, this approximation would correspond to neglecting the collision term in the equation for the intensity, to solve this independently and use the result as a source to the equation for polarization. This approach is consistent since, as we will see in Sec. IV, the polarization generated is very small so that we can neglect the backreaction of polarization on intensity.

${ }^{16}$ The velocity of galaxies is not biased $v(z, \mathbf{e})=v_{\mathrm{CDM}}(z, \mathbf{e})$.
} 


\section{A. The relation between intensity and the background energy density}

As discussed in [56], if we want to describe an inhomogeneous background, a useful quantity is the energy density of the background in a given direction, which is quadratic in the signal, does not depend on phases and has a nonvanishing correlation function:

$$
\Omega_{\mathrm{GW}}(\mathbf{e}) \equiv \frac{1}{\rho_{c}} \frac{d^{2} \rho_{\mathrm{GW}}}{d^{2} \mathbf{e}}(\mathbf{e}),
$$

where $\rho_{c}$ is the critical density of the universe and $d^{2} \rho_{\mathrm{GW}} / d^{2} \mathbf{e}$ is the energy density of the background in the solid angle $d^{2} \mathbf{e}$ around $\mathbf{e}$. It is useful to introduce the dimensionless energy density per unit of logarithmic frequency, as

$$
\Omega_{\mathrm{GW}}(\mathbf{e}) \equiv \int_{0}^{+\infty} d \log f \Omega_{\mathrm{GW}}(\mathbf{e}, f) .
$$

It follows that

$$
\Omega_{\mathrm{GW}}(\mathbf{e}, f)=\frac{f}{\rho_{c}} \frac{d^{3} \rho_{\mathrm{GW}}}{d^{2} \mathbf{e} d f}(\mathbf{e}, f),
$$

where $d^{3} \rho_{\mathrm{GW}}$ is the energy density of the background in the solid angle $d^{2} \mathbf{e}$ around $\mathbf{e}$ and in the frequency bin around $f$. Using the standard expression for the energy density in terms of the wave amplitude, see e.g., [79], and recalling that the definition of energy requires an average over several periods of the wave, we find

$$
\Omega_{\mathrm{GW}}(\mathbf{e}, f)=\frac{c^{2}}{4 G \rho_{c}} \frac{1}{T_{\mathrm{O}}} f^{3} \sum_{A}\left|\tilde{h}_{A}(f, \mathbf{e})\right|^{2},
$$

where the quantity $T_{\mathrm{O}}$ comes from the time average and represents the period of observation of the detector. ${ }^{17}$ The derivation of this result can be found in [56]. Using the definition of intensity, Eq. (2.21), we find

$$
\Omega_{\mathrm{GW}}(\mathbf{e}, f)=\frac{c^{2}}{4 G \rho_{c}} \frac{1}{T_{\mathrm{O}}} f^{3} I(\mathbf{e}, f) .
$$

\section{B. Radiation incident on a target}

We use the Boltzmann approach described in Appendix B to compute the energy density at a point

\footnotetext{
${ }^{17}$ The dependence on $T_{\mathrm{O}}$ may appear strange at first, but note that for a continuous background signal centered around a frequency $f$ with width $\Delta f$ one has for $T_{\mathrm{O}} \gg 1 / f$, $\int_{0}^{T_{\mathrm{O}}}\left|h_{A}(t)\right|^{2} d t \propto T_{\mathrm{O}} \simeq \int\left|h_{A}\left(f^{\prime}\right)\right|^{2} d f^{\prime} \simeq \Delta f\left|h_{A}(f)\right|^{2}$ and hence $\left|h_{A}(f)\right|^{2} \propto T_{\mathrm{O}}$. Hence the ratio $\frac{1}{T_{\mathrm{O}}} I(\mathbf{e}, f)$ is independent of $T_{\mathrm{O}}$. Of course for a short burst, averaging over a long time period $T_{\mathrm{O}}$ is not useful as it dilutes the signal. The adequate choice of $T_{\mathrm{O}}$ for this case is of course the duration of the signal.
}

$\left(\eta_{1}, \mathbf{x}_{1}\right)$ of the spacetime, from a direction $\mathbf{e}^{\prime}$ (see Fig. 1 for a representation of the situation under study). We neglect collisions. For a cosmological background we find

$$
\bar{\Omega}_{\mathrm{GW}}\left[\eta_{1}, f_{1}\right]=\frac{a\left(\eta_{i}\right)^{4}}{a\left(\eta_{1}\right)^{4}} \bar{\Omega}_{\mathrm{GW}}\left[\eta_{i}, f_{i}\right]
$$

and

$$
\begin{aligned}
\frac{\delta \Omega_{\mathrm{GW}}}{\bar{\Omega}_{\mathrm{GW}}}\left[\eta_{1}, \mathbf{x}_{1}, f_{1}, \mathbf{e}^{\prime}\right]= & \frac{\delta \Omega_{\mathrm{GW}}}{\bar{\Omega}_{\mathrm{GW}}}\left[\eta_{i}, \mathbf{x}_{2}, f_{i}\right] \\
& -4\left\{\phi\left[\eta_{1}, \mathbf{x}_{1}\right]-\phi\left[\eta_{i}, \mathbf{x}_{2}\right]\right. \\
& \left.-\mathbf{e}^{\prime} \cdot \mathbf{v}\left[\eta_{1}, \mathbf{x}_{1}\right]+\mathbf{e}^{\prime} \cdot \mathbf{v}\left[\eta_{i}, \mathbf{x}_{2}\right]\right\},
\end{aligned}
$$

where, to shorten the notation we have defined $\mathbf{x}_{2} \equiv \mathbf{x}_{1}+$ $\mathbf{e}^{\prime}\left(\eta_{1}-\eta^{\prime}\right)$. For an astrophysical background, keeping the leading term in the perturbation part in Eq. (B20)

$$
\begin{aligned}
\Omega_{\mathrm{GW}}\left[\eta_{1}, \mathbf{x}_{1}, \mathbf{e}^{\prime}, f_{1}\right]= & \frac{f_{1}}{4 \pi \rho_{c}} \int_{0}^{\eta_{1}} d \eta^{\prime} a^{4}\left(\eta^{\prime}\right) \bar{n}_{\mathrm{G}}\left(\eta^{\prime}\right) \\
& \times\left[1+\delta_{\mathrm{G}}\left[\eta^{\prime}, \mathbf{x}_{2}\right]\right] \\
& \times \int d \vartheta_{\mathrm{G}} \mathcal{L}_{\mathrm{G}}\left[\frac{1+z\left(\eta^{\prime}, \mathbf{x}_{2}\right)}{1+z\left(\eta_{1}, \mathbf{x}_{1}\right)} f_{1}, \vartheta_{\mathrm{G}}\right] .
\end{aligned}
$$

This results coincides with the one obtained in $[55,56]$. In Eq. (3.12), $\bar{n}_{\mathrm{G}}$ denotes the average density of galaxies, the galaxy overdensity is $\delta_{\mathrm{G}} \equiv b \delta_{\mathrm{m}}$ where $b$ is the bias function, $\delta_{\mathrm{m}}$ is the matter overdensity and $\mathcal{L}_{\mathrm{G}}$ is the effective luminosity of GW of a galaxy, per units of emitted frequency $f_{\mathrm{G}}=\left(1+z\left(\eta^{\prime}, \mathbf{x}_{2}\right)\right) /\left(1+z\left(\eta_{1}, \mathbf{x}_{1}\right)\right) f_{1}$ and $\vartheta_{\mathrm{G}}$ are a set of parameters effectively describing a given galaxy (mass, metallicity, etc.). The intensity corresponding to Eqs. (3.10)-(3.12) can be computed using Eq. (3.9).

We observe that the effective luminosity of a galaxy has been introduced in [55]: in the galaxy rest-frame, it represents the sum of the luminosity of gravitational waves of the various astrophysical sources contained in the galaxy, averaged with the distribution function of their peculiar velocity. As shown in [55], at linear order in the peculiar velocities, the effects of the peculiar motion of a source in its host galaxy can be neglected on average. The relation between the effective luminosity and the strain emitted can be found in Eqs. (79) and (80) of [56].

\section{Final result for the polarization tensor}

We now rewrite the formal result for the polarization tensor, Eq. (2.71), in terms of the building blocks already computed. The visibility function is defined in Eq. (2.13) and for small optical depth $\tau \ll 1$ it can be approximated as 


$$
\mathscr{V}\left(\eta_{0}, \eta_{1}\right) \simeq-\frac{\partial}{\partial \eta_{1}} \tau\left(\eta_{1}\right) \equiv-\dot{\tau}\left(\eta_{1}\right)
$$

where the derivative of the optical depth is given by Eq. (2.14). In Eq. (2.71) for the polarization tensor we found the intensity in terms of the background energy density by using Eq. (3.9) and we can use it to give us

$$
\bar{I}\left[\eta_{0}, \mathbf{x}_{0}, \mathbf{e}, f\right]=\frac{4 G \rho_{c}}{c^{2}} T_{\mathrm{O}} f^{-3} \bar{\Omega}_{\mathrm{GW}}\left[\eta_{0}, \mathbf{x}_{0}, \mathbf{e}, f\right],
$$

and

$$
\begin{aligned}
\delta I\left[\eta_{1}, \mathbf{x}_{1}, \mathbf{e}^{\prime}, f_{1}\right]= & \frac{4 G \rho_{c}}{c^{2}} T_{\mathrm{O}} \bar{f}_{1}^{-3} \bar{\Omega}_{\mathrm{GW}}\left[\eta_{1}, \mathbf{x}_{1}, f_{1}\right] \\
& \times\left[\frac{\delta \Omega_{\mathrm{GW}}}{\bar{\Omega}_{\mathrm{GW}}}\left[\eta_{1}, \mathbf{x}_{1}, \mathbf{e}^{\prime}, f_{1}\right]\right. \\
& \left.-3 \frac{\delta f_{1}}{\bar{f}_{1}}\left[\eta_{1}, \mathbf{x}_{1}, \mathbf{e}^{\prime}\right]\right]
\end{aligned}
$$

where we use that $f_{1}=\left(1+z\left(\eta_{1}, \mathbf{x}_{1}, \mathbf{e}^{\prime}\right)\right) f$ and split the frequency into a background and a perturbation part $f_{1}=\bar{f}_{1}+\delta f_{1}{ }^{18}$ In a cosmological framework, the perturbation of frequency gives a subleading contribution to Eq. (3.15) and for simplicity we neglect it in the following. At first order in perturbations the polarization tensor describing polarization generated by scattering off a given type (i) of targets is then given by

$$
\begin{aligned}
& \mathcal{P}_{a b}^{(i)}\left(\eta_{0}, \mathbf{x}_{0}, \mathbf{e}, f\right) \\
& =-\frac{\int_{0}^{\eta_{0}} d \eta_{1} \dot{\tau}^{(i)}\left(\eta_{1}\right) \int d^{2} \mathbf{e}^{\prime} \delta \Omega_{\mathrm{GW}}\left[\eta_{1}, \mathbf{x}_{1}, \mathbf{e}^{\prime}, f_{1}\right] \mathcal{S}_{a b}\left(\mathbf{e}, \mathbf{e}^{\prime}\right)}{2 \bar{\Omega}_{\mathrm{GW}}\left[\eta_{0}, \mathbf{x}_{0}, \mathbf{e}, f\right]},
\end{aligned}
$$

with

$$
\dot{\tau}^{(i)}\left(\eta_{1}\right)=-a\left(\eta_{1}\right) n_{s}^{(i)}\left(\eta_{1}\right) \sigma_{s}^{(i)},
$$

where $n_{s}$ is the physical number density of scattering centers of type $i$ (i.e., stellar mass black holes, super massive black hole ...) and $\sigma_{s}^{(i)}$ is the corresponding integrated cross section. Note that we are assuming that the objects causing diffraction have an isotropic distribution. Taking into account the angular dependence of the density of targets, $n_{s}^{(i)}\left(\eta_{1}, \mathbf{e}\right)$, is a straightforward generalization of our framework; one just needs to replace the optical depth in Eq. (3.17) with a direction dependent one $\tau\left(\eta_{1}\right) \rightarrow \tau\left(\eta_{1}, \mathbf{e}\right)$.

Considering only incoherent scattering, the total polarization is just the sum of the polarization generated by all the different scattering events (see Sec. IV D). ${ }^{19}$ The total polarization tensor is therefore given by

$$
\begin{aligned}
\mathcal{P}_{a b}^{\mathrm{tot}}\left(\eta_{0}, \mathbf{x}_{0}, \mathbf{e}, f\right) & =\sum_{i} \mathcal{P}_{a b}^{(i)}\left(\eta_{0}, \mathbf{x}_{0}, \mathbf{e}, f\right) \\
& =\frac{\int_{0}^{\eta_{0}} d \eta_{1} \sum_{i}\left(n_{s}^{(i)}\left(\eta_{1}, \mathbf{e}\right) \sigma_{s}^{(i)}\right) a\left(\eta_{1}\right) \int d^{2} \mathbf{e}^{\prime} \delta \Omega_{\mathrm{GW}}\left[\eta_{1}, \mathbf{x}_{1}, \mathbf{e}^{\prime}, f_{1}\right] \mathcal{S}_{a b}\left(\mathbf{e}, \mathbf{e}^{\prime}\right)}{2 \Omega_{\mathrm{GW}}\left[\eta_{0}, \mathbf{x}_{0}, \mathbf{e}, f\right]}
\end{aligned}
$$

This polarization tensor is a function of the energy density of the GW background which is given by Eqs. (3.10)(3.12) in terms of matter and metric perturbations. Polarization is therefore a stochastic quantity which can be characterized statistically in terms of its two-point function, like the energy density of the GW background in [55]. Moreover, it will cross-correlate with GW energy density and with other cosmological probes such as the galaxy distribution and weak lensing. We will investigate these aspects in future work.

We have assumed that the flux of the radiation emitted by a given object is unpolarized. For a cosmological background this is a very good assumption since we expect

\footnotetext{
${ }^{18}$ Perturbations of frequency are proportional to perturbations of redshift $\delta f_{1} / \bar{f}_{1}=\delta z /(1+\bar{z})$.

${ }^{19}$ In Sec. IV D we will show that the typical separation between scatterers (massive structures) is such that for the frequency range of present and planned GW experiments, multiscattering can be treated as incoherent.
}

that + and $\times$ polarization are produced with equal probability. An astrophysical background is given by the incoherent superposition of signals with random distribution of polarization. The average polarization of the background produced at a given redshift $z$ is therefore vanishing and the variance is proportional to the inverse of the number of sources at that redshift. Moreover, as we will see in Sec. IV, the polarization generated by scattering is much smaller than the intensity.

If an initial polarization is sufficiently small, we can just sum it to the polarization generated by interaction with matter. On the other hand, if the initial polarization is sizable, one can no longer neglect the backreaction of polarization on intensity. In this case, in our framework, one needs to go one step further and compute the intensity a background acquires after scattering and compute the new Stokes parameter considering the total intensity in expressions Eqs. (2.66)-(2.69). The procedure can be reiterated. A more rigorous approach consists in solving a set of Boltzmann equations for intensity and polarization with a 
collision term which can be derived considering the angular dependence of the cross section (2.9). This can be done by determining a scattering matrix for all Stokes parameters exactly as for the CMB, see e.g., [78]. In the present approach, however, we assume polarization to be very small and neglect its backreaction on intensity.

\section{AN ESTIMATE OF THE POLARIZED GW BACKGROUND}

We now want to derive an order of magnitude estimate of the polarization parameter $P$, introduced in Eq. (2.26) that a GW background acquires due to interactions with matter. We assume that scattering off massive structures is the only source of polarization. If the GW flux is initially unpolarized, the amount of polarization (averaged over directions) measured by the observer is proportional to the probability of scattering and to the amount of anisotropies of the radiation incident on a scattering center. Using the results of the previous sections, we can derive an estimate of the amount of polarization a flux of gravitons produced at $z$ and received today as

$$
P(z)=\frac{\delta \Omega_{\mathrm{GW}}}{\bar{\Omega}_{\mathrm{GW}}} \times\left[1-e^{-\tau(z)}\right]
$$

where the optical depth $\tau$ is defined in Eq. (2.10). For comparison, for the $\mathrm{CMB}$, assuming that all the polarization is generated by reionization and $\tau_{\text {rei }}=0.08$, one finds

$$
P_{\mathrm{CMB}}^{\mathrm{rei}}=\frac{\delta T}{\bar{T}} \times\left[1-e^{-\tau\left(z_{\mathrm{rei}}\right)}\right] \simeq 10^{-6}
$$

Two main ingredients enter Eq. (4.1): the fractional anisotropy of a GW background and the optical depth. This last ingredient depends on the density of scattering centers and on the effective cross section of the scattering process. In this section we derive an order of magnitude estimate for the optical depth for different types of astrophysical objects acting as scattering centers. The optical depth will be a function of the GW frequency $1 / \lambda$. For future reference, in Table I we list the wavelength range of (some) current and planned GW experiments.

TABLE I. Range of wavelengths of different GW experiments in units of parsec [pc]. We denote the wavelength that we will take as a reference for order of magnitude estimates by $\lambda_{M}$.

\begin{tabular}{lrc}
\hline \hline Experiment & $\lambda_{\mathrm{O}}[\mathrm{pc}]$ & $\lambda_{M}[\mathrm{pc}]$ \\
\hline LIGO & $10^{-11}-10^{-9}$ & $10^{-10}$ \\
LISA & $10^{-9}-10^{-5}$ & $10^{-7}$ \\
PTA & $10^{-5}-10^{-1}$ & $10^{-3}$ \\
\hline \hline
\end{tabular}

\section{A. Diffraction by compact and extended objects}

The metric associated to a compact massive object with $r_{s}=2 M G$ is the Schwarzschild metric. In Schwarzschild coordinates $(t, r, \theta, \phi)$ the metric can be written as

$$
\begin{aligned}
\bar{g}_{\mu \nu} d x^{\mu} d x^{\mu}= & -(1+2 \Phi) d t^{2}+(1+2 \Phi)^{-1} d r^{2} \\
& +r^{2} d \theta^{2}+r^{2} \sin \theta^{2} d \phi^{2},
\end{aligned}
$$

with $\Phi=-G M / r$. In Lorenzian coordinates one has

$$
\begin{aligned}
\bar{g}_{\mu \nu} d x^{\mu} d x^{\mu}= & -\left(\frac{1-M G /(2 \rho)}{1+M G /(2 \rho)}\right)^{2} d t^{2} \\
& +\left(1-\frac{M G}{2 \rho}\right)^{4}\left(d \rho^{2}+\rho^{2} d \theta^{2}+\rho^{2} \sin \theta^{2} d \phi^{2}\right),
\end{aligned}
$$

with

$$
r=\rho\left(1-\frac{M G}{2 \rho}\right)^{2}
$$

For such a spacetime the Kretschmann scalar is given by

$$
\sqrt{\bar{K}}=\sqrt{\bar{R}_{\mu \alpha \nu \beta} \bar{R}^{\mu \alpha \nu \beta}}=2 \sqrt{3} \frac{r_{s}}{r^{3}} .
$$

Using Eq. (2.4) giving the regime of validity of geometric optics, we find that wavelike effects are expected in a region

$$
r_{s} \leq r \leq R_{\lambda}
$$

with

$$
R_{\lambda} \equiv\left(2 \sqrt{3} \lambda^{2} r_{s}\right)^{1 / 3}
$$

The condition (4.7) can be satisfied if $r_{s} \leq R_{\lambda}$, which up to factors of order unity is equivalent to $r_{s}<\lambda$. Note that one needs to include a factor of redshift $\lambda_{\mathrm{O}}=(1+z) \lambda$ so that

$$
\frac{\lambda_{\mathrm{O}}}{\mathrm{pc}} \geq 10^{-13} \beta(1+z),
$$

where $\beta=M / M_{\odot}$. We recall that for stellar mass black holes $\beta \in[5-50]$ while for a super massive black hole $\beta \in\left[10^{4}-10^{9}\right]$. Choosing typical values of $\beta$ for the two cases, taking into account that most astrophysical sources are located at $z \sim 1-2$ and using the values of $\lambda_{\mathrm{O}}$ tested by present experiments (see Table I) we find that for solar mass $\mathrm{BH}$, diffraction effects are present in all frequency bands, while for supermassive $\mathrm{BH}$ diffraction is relevant only in the PTA and (partially) in the LISA bands.

Primordial black holes have a much broader redshift distribution. For a given frequency band and average mass 
$\beta$, using Eq. (4.9), one can derive a condition on the redshift range where polarization is generated through diffusion. ${ }^{20}$

For a spherically symmetric extended object with radius $R$, mass $M$ and constant density $\rho$, the gravitational potential is given by

$$
\Phi= \begin{cases}-\frac{G M}{r}, & r>R \\ -G M \frac{\left(3 R^{2}-r^{2}\right)}{2 R^{3}}, & r \leq R\end{cases}
$$

The corresponding metric can be written by making use of Lorenzian coordinates as

$$
\begin{aligned}
g_{\mu \nu} d x^{\mu} d x^{\nu}= & -e^{2 \Phi(\rho)} d t^{2}+e^{-2 \Phi(\rho)}\left(d \rho^{2}+\rho^{2} d \theta^{2}\right. \\
& \left.+\rho^{2} \sin \theta^{2} d \phi^{2}\right) .
\end{aligned}
$$

Note that, to first order in $\Phi$, the difference between Schwarzschild and Lorentzian coordiates can be neglected. At leading order in $r_{s}$, one has (in Schwarzschild coordinates)

$$
\bar{R}_{\mu \alpha \nu \beta} \bar{R}^{\mu \alpha \nu \beta}= \begin{cases}12 \frac{r_{s}^{2}}{r^{6}}, & r>R, \\ 15 \frac{r_{s}^{2}}{R^{6}}, & r \leq R .\end{cases}
$$

The fact that the Kretschmann scalar is discontinuous at $r=R$ is not surprising since also the density and therefore the Ricci tensor jump at this value of $r$. Outside the object, wavelike effects are present in a region

$$
R<r \leq R_{\lambda},
$$

with $R_{\lambda}$ defined in Eq. (4.8). Writing $R=\alpha R_{\odot}, M=\beta M_{\odot}$ and setting $\gamma=\beta / \alpha^{3}$, (4.13) can be verified if $R \leq R_{\lambda}$, i.e., if

$$
\lambda_{\mathrm{O}}>\frac{1}{\sqrt{\gamma}}(1+z) 10^{-6} \mathrm{pc}
$$

Inside the object, corrections to geometric optics are present for $\sqrt{15} r_{s} / R^{3}>\lambda^{-2}$ which also reproduces roughly condition (4.14). Considering the values of $\gamma$ listed in Table $\mathrm{II}^{21}$ and the typical wavelengths for different experiments are given in Table I, we find that most stars produce

\footnotetext{
${ }^{20}$ For example, assuming that all primordial black holes have $M \sim M_{\odot}$, from Eq. (4.9) and using the average value of wavelength of different experiments (see Table I), we find that in the LIGO, LISA and PTA band polarization effects are coming from redshift up to $z \simeq 10^{3}, z \simeq 10^{6}$ and $z \simeq 10^{10}$ respectively. As $\lambda_{O}$ scales like $\beta(1+z)$, this redshift scales like $M^{-1}$.

${ }^{21}$ In Table II we consider the average values of masses for main sequence stars, with $\beta \leq 40$. More massive main sequence stars (with mass up to $\sim 200 M_{\odot}$ ) exist, but are quite rare, see e.g., [80]. For comparison, we included the range of average radii and masses of super giants, even if this is a very short lived stage of stellar evolution.
}

TABLE II. Masses, radii and compactness of different types of stars [81-85].

\begin{tabular}{lccc}
\hline \hline Category & $\alpha=R / R_{\odot}$ & $\beta=M / M_{\odot}$ & $\gamma=\beta / \alpha^{3}$ \\
\hline Main sequence & $0.1-20$ & $0.1-40$ & $0.01-45$ \\
White dwarfs & $0.003-0.03$ & $0.17-1.33$ & $6 \times 10^{4}-5 \times 10^{7}$ \\
Super giants & $30-1500$ & $8-12$ & $10^{-9}-10^{-4}$ \\
\hline \hline
\end{tabular}

diffraction effects in the PTA band, in addition white dwarfs give contributions in the LISA band while wavelengths of the LIGO band are not affected by diffraction effects from extended objects.

\section{B. Integrated cross section}

We have found that for both compact and extended objects, wave-like effects lead to polarization occurring in a region defined by the conditions (4.7) and (4.13) respectively. These conditions fix an upper bound on the impact parameter $b_{\max }=R_{\lambda}$ and correspondingly a lower bound on the deflection angle

$$
\theta_{\min }=\frac{2 r_{s}}{b_{\max }} \simeq\left(\frac{r_{s}}{\lambda}\right)^{2 / 3}
$$

Moreover, when dealing with a compact object, absorption occurs for $b<r_{s}$ and this defines a maximum value for the scattering angle $\theta_{\max }=2$. This bound is absent in the case of an extended object. The differential cross section (2.9) summed over polarizations is given by Eq. (2.5) for $s=2$. The total cross section can be found by integrating (2.5) over angles with $\theta \in\left[\theta_{\min }, \theta_{\max }\right]$. The result is

$$
\begin{aligned}
\sigma\left(\lambda, r_{s}\right)= & \frac{2 \pi}{3}(G M)^{2}[-111 \cos \theta-6 \cos (2 \theta)-\cos (3 \theta) \\
& \left.-48 \sin ^{-2}\left(\frac{\theta}{2}\right)-384 \log \left(\sin \frac{\theta}{2}\right)\right]_{\theta_{\min }}^{\theta_{\max }}
\end{aligned}
$$

For $r_{s} \ll \lambda, \theta_{\min }$ is very small and the expression in square brackets can be approximated by the fourth term, which yields

$$
\sigma\left(\beta, \lambda_{\mathrm{O}}, z\right) \simeq 10^{-7} \beta^{2 / 3}\left(\frac{\lambda_{\mathrm{O}}}{\mathrm{pc}}\right)^{4 / 3}(1+z)^{-4 / 3} \mathrm{pc}^{2},
$$

where we used $\lambda_{\mathrm{O}}=(1+z) \lambda$. This approximation is always well justified for stellar mass black holes and extended objects: the minimum wavelength we have access to is $\lambda \simeq 10^{-11} \mathrm{pc}$ and considering $r_{s}\left(M_{\odot}\right) \simeq 10^{-13} \mathrm{pc}$, we have $r_{s}\left(M_{\odot}\right) / \lambda \leq r_{s}\left(M_{\odot}\right) / \lambda_{\min } \simeq 10^{-2}$. For supermassive black holes, we can have $\lambda \simeq r_{s}$ and the full expression (4.16) for the integrated cross section must be used. We will however make use of (4.17) for a first estimate (the error is a few percent). 
We now consider the case of an extended object and an impact parameter smaller than the size of the object $b<R$. We effectively describe wave-like effects in a region (4.14) inside the object as a process of scattering of GW off a spherical target of radius $b$ and mass $M_{b}=M(b / R)^{3}$. The scattering angle for such a process is fixed and given by $\theta \approx 2 r_{s} / b$. In this case the differential cross section (2.5) can be approximated as

$$
\frac{d \sigma}{d \Omega} \approx 8\left(G M_{b}\right)^{2}\left(\frac{b}{r_{s}}\right)^{4}
$$

Writing, as before, $R=\alpha R_{\odot}$ and $M=\beta M_{\odot}$, the expression above can be simplified to

$$
\frac{d \sigma}{d \Omega} \approx 2 \times 10^{-6} \frac{\alpha^{4}}{\beta^{2}}\left(\frac{b}{R}\right)^{10} \mathrm{pc}^{2} \leq 2 \times 10^{-6} \frac{\alpha^{4}}{\beta^{2}} \mathrm{pc}^{2} .
$$

The integrated cross section is (the integration over angles is simply an integration over $\phi$ and gives a factor $2 \pi$ )

$$
\sigma(\alpha, \beta, b) \simeq 10^{-5} \frac{\alpha^{4}}{\beta^{2}}\left(\frac{b}{R}\right)^{10} \mathrm{pc}^{2} .
$$

Equations (4.17) and (4.20) are the final results of this section.

\section{Optical depth}

For scattering off compact objects and extended objects with $b>R$, the integrated cross section Eq. (4.17), is redshift-dependent. For this case, the optical depths, Eq. (2.10) for scattering in a matter dominated universe can be rewritten as

$$
\tau(z) \simeq 6 n \bar{\sigma}\left(H_{0} \sqrt{\Omega_{m}}\right)^{-1}\left[(1+z)^{1 / 6}-1\right],
$$

where $n$ is the comoving number density of targets and $\bar{\sigma}$ is the redshift-independent part of the cross section (4.17),

$$
\bar{\sigma} \equiv 10^{-7} \beta^{2 / 3}\left(\frac{\lambda_{\mathrm{O}}}{\mathrm{pc}}\right)^{4 / 3} \mathrm{pc}^{2} .
$$

Analogously, for scattering taking place in a radiation dominated universe we have

$\tau(z) \simeq 3 n \bar{\sigma}\left(H_{0} \sqrt{\Omega_{r}}\right)^{-1}\left[\frac{1}{\left(1+z_{\mathrm{eq}}\right)^{1 / 3}}-\frac{1}{(1+z)^{1 / 3}}\right]$,

where $z_{\text {eq }}$ is the redshift at equality i.e., equal energy density in matter and radiation. We will make use of this last expression only when considering the case of primordial black holes. For scattering off extended objects with $b<R$, the integrated cross section (4.20) does not depend on redshift. The optical depth for this case and for $z \ll z_{\text {eq }}$ can be written as

$$
\tau(z) \simeq n \sigma\left(H_{0} \sqrt{\Omega_{m}}\right)^{-1} \frac{2}{3}\left[(1+z)^{3 / 2}-1\right],
$$

with $\sigma$ given by Eq. (4.20). In the following we compute the optical depth for scattering off different types of astrophysical objects, namely stellar mass black holes, supermassive black holes, primordial black holes and extended objects (stars).

The energy density of baryons in the observed universe is given by

$$
\rho_{B}=\rho_{c} \Omega_{B} \simeq M_{\odot} \mathrm{kpc}^{-3} .
$$

Only about $10 \%$ of the total baryonic matter has collapsed sufficiently to form stars and galaxies; the remaining 90\% makes up the gas in clusters and the intergalactic medium. Denoting the number of stars, stellar mass black holes $(\mathrm{BH})$ and supermassive black holes $(\mathrm{SBH})$ in a galaxy by $N_{*}, N_{\mathrm{BH}}$ and $N_{\mathrm{SBH}}$, we expect $N_{\mathrm{SBH}}=10^{-8} N_{\mathrm{BH}}=$ $10^{-8}\left(10^{-3} N_{*}\right)$. Then, assuming that all stars and stellar mass black holes have mass equal to the solar mass and assuming that sources are homogeneously distributed (i.e., we neglect the presence of structures in this first step), we find that the comoving density of stars is ${ }^{22}$

$$
n_{*} \approx \frac{\rho_{*}}{M_{\odot}}=0.1 \mathrm{kpc}^{-3},
$$

and for stellar mass black holes and supermassive black holes, respectively

$$
\begin{gathered}
n_{\mathrm{BH}} \simeq 10^{-4} \mathrm{kpc}^{-3}, \\
n_{\mathrm{SBH}} \simeq 10^{-12} \mathrm{kpc}^{-3} .
\end{gathered}
$$

Using Eq. (4.17) in Eq. (4.21) we obtain

$$
\tau_{\mathrm{BH}}\left(z, \lambda_{\mathrm{O}}\right) \simeq 10^{-9}\left(\frac{\lambda_{\mathrm{O}}}{\mathrm{pc}}\right)^{4 / 3}\left[(1+z)^{1 / 6}-1\right] .
$$

Analogously, for supermassive black holes

$\tau_{\mathrm{SBH}}\left(z, \lambda_{\mathrm{O}}, \beta\right) \simeq 10^{-17} \beta^{2 / 3}\left(\frac{\lambda_{\mathrm{O}}}{\mathrm{pc}}\right)^{4 / 3}\left[(1+z)^{1 / 6}-1\right]$,

with $\beta \in\left[10^{4}, 10^{9}\right]$.

Next, we consider the possibility that primordial black holes $(\mathrm{PBH})$ represent a fraction $q$ of the dark matter energy density, i.e.,

\footnotetext{
${ }^{22} \mathrm{We}$ are making the assumption $0.1 \rho_{B}=M_{\odot}\left(n_{*}+n_{\mathrm{BH}}+\right.$ $\left.\beta n_{\mathrm{SBH}}\right) \approx M_{\odot} n_{*}$ where $\beta \in\left[10^{4}, 10^{9}\right]$.
} 


$$
\Omega_{\mathrm{PBM}}=q \Omega_{\mathrm{CDM}},
$$

with $q \in[0,1]$. The comoving number density of primordial black holes can be estimated as

$$
n_{\mathrm{PBH}}=\rho_{c} \frac{\Omega_{\mathrm{CDM}}}{M_{\mathrm{PBH}}} q .
$$

Writing $M_{\mathrm{PBH}}=\beta M_{\odot}$ with $\beta<10^{3}$ this yields

$$
n_{\mathrm{PBH}} \simeq \frac{q}{\beta}(\mathrm{kpc})^{-3} .
$$

PBH have a broad redshift distribution, which extends up to very high redshift, see e.g., [86] for a recent review. It follows that a cosmological background of GW radiation (produced during or after inflation) can scatter in a broad redshift range. Inserting Eqs. (4.17) and (4.33) in (4.23), we find

$\tau_{\mathrm{PBH}}^{\mathrm{cosmo}}\left(z, \lambda_{\mathrm{O}}, \beta\right) \simeq \frac{q}{\beta^{1 / 3}} 10^{-4}\left(\frac{\lambda_{\mathrm{O}}}{\mathrm{pc}}\right)^{4 / 3} \frac{1}{\left(1+z_{\mathrm{eq}}\right)^{1 / 3}}$.

For an astrophysical background of GW, we use (4.21) to find

$\tau_{\mathrm{PBH}}^{\mathrm{astro}}\left(z, \lambda_{\mathrm{O}}, \beta\right) \simeq \frac{q}{\beta^{1 / 3}} 10^{-5}\left(\frac{\lambda_{\mathrm{O}}}{\mathrm{pc}}\right)^{4 / 3}\left[(1+z)^{1 / 6}-1\right]$.

We turn now to the case of GW scattering off a distribution of stars. The condition to have diffusion is given by Eq. (4.14). We use the results in Tables I and II: diffraction occurs for all the physical values of $\gamma$ in the PTA band, in the LISA band a contribution comes only from scattering off white dwarfs while no effect is present in the LIGO band. Assuming that all the stars have Solar mass, the comoving density of stars is given by Eq. (4.26). Then, in the PTA, replacing (4.26) and (4.17) in (4.21) and choosing $\beta=1=\alpha$, we find

$$
\tau_{* \text { out }}^{\mathrm{PTA}}\left(z, \lambda_{\mathrm{O}}\right) \simeq 10^{-6}\left(\frac{\lambda_{\mathrm{O}}}{\mathrm{pc}}\right)^{4 / 3}\left[(1+z)^{1 / 6}-1\right]
$$

for scattering with impact parameter bigger than the size of the object. For scattering off extended objects with impact parameter smaller than the object size, the (effective) cross section describing such a process is given in Eq. (4.20). Then, in the PTA band, assuming $\beta=1=\alpha$ and plugging (4.20) and (4.26) in (4.24), we have

$$
\tau_{* \text { in }}^{\mathrm{PTA}}(z, b) \simeq 10^{-5}\left(\frac{b}{R_{\odot}}\right)^{10}\left[(1+z)^{3 / 2}-1\right] .
$$

Assuming the impact parameter $b$ to be distributed uniformly in $\left[0, R_{\odot}\right]$ and choosing, for the estimate, the average value $\langle b\rangle=R_{\odot} / 2$, we find

$$
\tau_{* \text { in }}^{\mathrm{PTA}}(z) \simeq 10^{-8}\left[(1+z)^{3 / 2}-1\right] .
$$

For the LISA band, denoting as $f_{\text {wd }}$ the fraction of stars in white dwarfs, we find

$\tau_{* \text { out }}^{\mathrm{LISA}}\left(z, \lambda_{\mathrm{O}}\right) \simeq 10^{-6} f_{\mathrm{wd}}\left(\frac{\lambda_{\mathrm{O}}}{\mathrm{pc}}\right)^{4 / 3}\left[(1+z)^{1 / 6}-1\right]$,

$\operatorname{and}^{23}$

$$
\tau_{* \text { in }}^{\mathrm{LISA}}(z) \simeq 10^{-8} f_{\mathrm{wd}}\left[(1+z)^{3 / 2}-1\right] .
$$

We emphasize that here we assume that astrophysical sources have an isotropic distribution. As we will see in the next Sec. IV D, the average distance between objects in a structure is such that the multi-scattering of gravitational waves off massive structures can be considered as incoherent and the total polarization generated through diffusion is the linear sum of polarization created off different scatterings. It follows that, as long as we are interested in deriving an estimate of the total polarization generated (averaged over directions at the observer position), neglecting the presence of structures is well justified.

\section{Coherent and incoherent scattering}

Let us consider a distribution of compact objects/stars, with density $n$ and mean distance $d=n^{-1 / 3}$ (i.e., $d$ is the average distance between objects) and masses $M_{i}$. If we have a wave with wavelength $\lambda$ incident on this distribution, depending on the relative size of $\lambda$ and $d$, the process has to be treated as either coherent or incoherent. To be specific we have that

(i) if $\lambda \gg d$ the scattering is coherent;

(ii) if $\lambda \ll d$ the scattering is incoherent.

If the scattering is coherent, then the multiscattering process can be treated as a single scattering off a target with mass $M_{\mathrm{tot}}=\sum_{i} M_{i}$. The total cross section is given by

$$
\frac{d \sigma}{d \Omega} \propto\left(\sum_{i} M_{i}\right)^{2} G^{2}[\ldots]
$$

where $[. .$.$] stays for some angular structure. If the scattering$ is incoherent, the total cross section is given by the linear sum of single cross sections

$$
\frac{d \sigma}{d \Omega} \propto \sum_{i}\left(M_{i} G\right)^{2}[\ldots]
$$

Let us consider a GW passing through a globular cluster. The number of stars in a globular cluster is $N_{*} \simeq 10^{4}-10^{5}$

\footnotetext{
${ }^{23}$ The local (midplan) mass density of white dwarfs is $\rho_{\mathrm{wd}} \simeq 0.0065 M_{\odot} \mathrm{pc}^{-3}$, see e.g., [87]. Considering that the average mass of stars is $\sim 0.5 M_{\odot}$ and comparing to the local density of stars, see e.g., [88], one obtains the local value $f_{\text {wd }} \sim 0.05$.
} 
TABLE III. Optical depth for scattering off different types of massive structures.

\begin{tabular}{lccc}
\hline \hline & PTA $\left(\lambda_{M}=10^{-3}\right)$ & LISA $\left(\lambda_{M}=10^{-7}\right)$ & LIGO $\left(\lambda_{M}=10^{-10}\right)$ \\
\hline$\tau_{\mathrm{BH}}$ & $10^{-14}$ & $10^{-19}$ & $10^{-23}$ \\
$\tau_{\mathrm{SBH}}$ & $\beta^{2 / 3} 10^{-22}$ & $\beta^{2 / 3} 10^{-27}$ & 0 \\
$\tau_{*, \text { in }}$ & $10^{-8}$ & $f_{\mathrm{wd}} 10^{-8}$ & 0 \\
$\tau_{*, \text { out }}$ & $10^{-11}$ & $f_{\mathrm{wd}} 10^{-16}$ & 0 \\
$\tau_{\mathrm{PBH}, \text { astro }}$ & $q \beta^{-1 / 3} 10^{-10}$ & $q \beta^{-1 / 3} 10^{-15}$ & $q \beta^{-1 / 3} 10^{-19}$ \\
$\tau_{\mathrm{PBH}, \text { cosmo }}$ & $q \beta^{-1 / 3} 10^{-9}$ & $q \beta^{-1 / 3} 10^{-14}$ & $q \beta^{-1 / 3} 10^{-18}$ \\
\hline \hline
\end{tabular}

and the (typical) radius of the cluster $R_{\mathrm{GC}} \simeq 10 \mathrm{pc}$. The average distance between objects is then given by $d_{\mathrm{GC}}=$ $n_{\mathrm{GC}}^{-1 / 3} \simeq\left(V_{\mathrm{GC}} / N_{*}\right)^{1 / 3} \simeq 1 \mathrm{pc}$ (where $V_{\mathrm{GC}}$ is the volume of the cluster). Comparing with the wavelength we have access to observationally (see Table I) we always have $d_{\mathrm{GC}}>\lambda_{\mathrm{O}}>\lambda$ : the multiscattering process can be treated as incoherent scattering (some coherence could be present in the low frequency part of the PTA range). If we repeat the same reasoning for a galaxy we get $d>d_{\mathrm{GC}}$ (objects are less densely distributed) and the same conclusion holds, except maybe close to the galactic center where scattering however is dominated by the central super massive black hole.

\section{E. The total amount of polarization}

For a given wavelength, the total amount of polarization produced by diffusion is given by the sum of the polarization produced from scattering off different types of massive structures. We recall that the condition to have diffusion effects in the vicinity of a compact object is given by Eq. (4.9) while the analogous condition for an extended object is (4.14). Using the results in Tables I and II, we find that in different frequency bands, different objects are causing diffraction effects which lead to polarization

(i) PTA band: solar mass BH, supermassive black holes, any type of star;

(ii) LISA band: solar mass BH, (some) (super)massive black hole, white dwarfs;

(iii) LIGO band: solar mass $\mathrm{BH}$.

The condition to have scattering off primordial black holes is more subtle since PBH have a broad redshift distribution. Considering a vanilla model where all PBH have the same mass $M=\beta M_{\odot}$, for a given observed frequency, the condition (4.9) gives the maximum redshift at which we can have diffusion and polarization generation. In particular, all solar mass $\mathrm{PBH}$ (or lighter) up to $z=z_{\mathrm{eq}}$ act as scattering centers and produce polarization of the background in the entire frequency range of present and planned GW observatories.

We can now work out an estimate for the polarization fraction (4.1) for the LIGO, LISA and PTA band. To do so, we introduce a number of simplifications. For the three cases, we choose $\lambda_{M}$ from Table I. Moreover, since most astrophysical sources are expected to be located around redshift $z=1-2$ (see e.g., [89]) we evaluate the visibility function at $z=1.5$. If primordial black holes represent a fraction $q$ of the total dark matter component, we distinguish two cases: scattering taking place in matter domination and in radiation domination. Obviously, only a background of cosmological origin can undergo the latter type of process. In this first approximation, we assume that the optical depth for scattering off PBH at early times is redshift independent. ${ }^{24}$ At late times we assume the baryonic matter distribution to follow the distribution of primordial black holes and we consider most of these targets to be located around $z=1.5$. Our results are summarized in Table III.

The total amount of polarization is given by the sum of the polarization generated by different scatterings, i.e., the total parameter (4.1) is given by

$$
P=\sum_{i} P_{i} \simeq \frac{\delta \Omega_{\mathrm{GW}}}{\bar{\Omega}_{\mathrm{GW}}} \sum_{i} \tau_{i}
$$

where $i$ labels different types of scatterers. In the last equality we have used that $\tau \ll 1$ and $\delta \Omega_{\mathrm{GW}} / \bar{\Omega}_{\mathrm{GW}}$ is the typical amount of anisotropies of a given component of the background. An estimate of this quantity as a function of redshift for a cosmological and astrophysical background can be found in Appendix C. For a cosmological background, in any frequency range, $\delta \Omega_{\mathrm{GW}} / \bar{\Omega}_{\mathrm{GW}} \sim 10^{-5}$. For an astrophysical background the result depends on frequency, on the astrophysical sources we consider and on redshift. In the LIGO band the background is dominated by the contribution from black hole mergers and at $z=1.5$ where we assume most of the sources producing scattering to be located, we have $\delta \Omega_{\mathrm{GW}} / \bar{\Omega}_{\mathrm{GW}} \sim 10^{-3}$. If we extrapolate the LIGO estimate of the astrophysical background to all frequencies we then have

\footnotetext{
${ }^{24}$ At earlier times, the optical depth has a mild redshift dependence, see Eq. (4.23) which we neglect.
} 


$$
\frac{\delta \Omega_{\mathrm{GW}}}{\bar{\Omega}_{\mathrm{GW}}} \simeq \begin{cases}10^{-3}, & \text { for astrophysical background } \\ 10^{-5}, & \text { for cosmological background }\end{cases}
$$

In the LIGO band the dominant contribution to polarization comes from diffusion off $\mathrm{PBH}$ and stellar mass black holes. The total amount of polarization generated is given by

$$
P_{\mathrm{LIGO}} \simeq \begin{cases}10^{-22}\left(q \beta^{-1 / 3}+10^{-4}\right), & \text { for astrophysical background } \\ 10^{-23}\left(q \beta^{-1 / 3}+10^{-5}\right), & \text { for cosmological background }\end{cases}
$$

In the LISA band, the dominant contribution to polarization generation comes from scattering off white dwarfs and the amount of polarization generated is given by

$$
P_{\text {LISA }} \simeq f_{\text {wd }} 10^{-11},
$$

for an astrophysical background while for a cosmological background, the result is suppressed by an additional factor $10^{-2}$. In the PTA band, the processes generated more efficiently polarization are scattering off PBH and extended objects. One finds

$$
P_{\mathrm{PTA}} \simeq \begin{cases}10^{-11}\left(1+0.01 q \beta^{-1 / 3}\right), & \text { for astrophysical background } \\ 10^{-13}\left(1+0.1 q \beta^{-1 / 3}\right), & \text { for cosmological background }\end{cases}
$$

To get a first order of magnitude estimate of the effect, we consider the simplest scenario of PBH with monochromatic mass distribution $M=M_{\odot}$ and $q=0.5$, still allowed by current observational bounds, and we extrapole the local value $f_{\mathrm{wd}}=0.05$ at extragalactic scales. The results are listed in Table IV.

We emphasize that the estimates (4.45), (4.46) and (4.47) for a cosmological background are robust since the prediction $\delta \Omega_{\mathrm{GW}} / \bar{\Omega}_{\mathrm{GW}} \sim 10^{-5}$ does not depend on astrophysical complications and on frequency (see Appendix C). For an astrophysical background, we are extrapolating the prediction for $\delta \Omega_{\mathrm{GW}} / \bar{\Omega}_{\mathrm{GW}}$ valid in the LIGO band to lower frequencies. To derive a more realistic estimate of $P$ for an astrophysical background in the PTA and LISA band, one has to repeat the analysis of [57] valid for the LIGO frequency band, and work out the amplitude of anisotropies at lower frequency, including contributions from different astrophysical sources. This analysis will be presented in [90].

TABLE IV. Polarization of an astrophysical and cosmological generated through diffusion off massive structures in different frequency bands. The estimate has been obtained under the assumptions of Sec. IVE: $\mathrm{PBH}$ with monochromatic mass distribution and $\beta=2 q=1$ and average fraction of white dwarfs equal to the local one.

\begin{tabular}{lccc}
\hline \hline & PTA & LISA & LIGO \\
$\left(\lambda_{M}=10^{-3}\right)$ & $\left(\lambda_{M}=10^{-7}\right)$ & $\left(\lambda_{M}=10^{-10}\right)$ \\
\hline$P_{\text {astro }}$ & $10^{-11}$ & $10^{-12}$ & $10^{-23}$ \\
$P_{\text {cosmo }}$ & $10^{-13}$ & $10^{-14}$ & $10^{-24}$ \\
\hline \hline
\end{tabular}

\section{CONCLUSIONS}

In this work, we have discussed the production of polarization of a stochastic GW background from diffusion by extended and compact astrophysical objects. We have provided a framework which can be used to compute the polarization tensor of a given component of the background, in any frequency range. The main ingredients of our approach are the following. We compute the integrated cross section for scattering off a given massive structure. Since the geometric optics approximation breaks down in a region of radius $R_{\lambda}$ around the scatterer, the impact parameter has an upper bound $b_{\max }=R_{\lambda}$ and with $b_{\max }$, the integrated cross section for a compact (extended) object depends on the mass (on the compactness) of the object and on the wavelength of the GW. As a consequence, the optical depth depends on the abundance of targets, on the properties of the target and on the wavelength. We have discussed that, for the wavelength range we have access to in present and planned GW experiments, the average separation between scattering centers is much larger than the wavelength, hence multiscattering can be studied as an incoherent sum of scattering processes. To compute the total polarization which the GW background acquires due to interaction with structures, it is therefore sufficient to sum the polarization generated by different types of scatterings.

In our framework, the back reaction of polarization on intensity is systematically discarded. More precisely, we compute the intensity of the GW background measured by a comoving observer at a given redshift and from a given direction, using the geometric optics approximation and we use this result to compute the polarization created from a scattering event. The geometric optics approximation is not 
suitable to describe the creation of polarization by interaction with matter: wave like effects represent a first order correction to the intensity while they give a zero order contribution to polarization. Since the polarization created by scattering is very suppressed with respect to the intensity, see Sec. IV, neglecting the back reaction of polarization on intensity is well justified.

We have also assumed that the flux of radiation at emission is unpolarized. As already discussed, this is a very good assumption for the case of a cosmological background, for which we expect that the two polarizations are generated with equal probability. If the initial (primary) polarization is sufficiently small, it can be simply added to the secondary one created during the propagation to the observer position, neglecting back reaction. A more rigorous approach consists in writing a set of Boltzmann equations for intensity and polarization, with a collision term that is coupling the system. Writing such a system is rather straightforward: one needs to use the results of Appendix B and derive the angular dependence of the collision term from the angular dependence of the differential cross section, Eq. (2.9). ${ }^{25}$

In Sec. IV we have derived an order of magnitude estimate for the polarization in different frequency bands for both astrophysical and cosmological GW backgrounds. In the LIGO band the dominant contribution to the creation of polarization comes from scattering off stellar mass black holes or PBH if they exist; in the LISA band scattering off white dwarfs dominates while in the PTA band from scattering off stars (or PBH if they exist) is most important. For an astrophysical background in the LIGO band, in the scenario in which primordial black holes have a monochromatic mass distribution with $M=M_{\odot}$ and constitute half of the total amount of dark matter, ${ }^{26}$ the amount of polarization created is suppressed with respect to (energy density) anisotropies by a factor $10^{-20}$ and $10^{-19}$ for an astrophysical and cosmological background, respectively. An enhancement of this result can be obtained in the case in which the mass distribution of $\mathrm{PBH}$ is not monochromatic

\footnotetext{
${ }^{25}$ However, an additional complication with respect to the case of the CMB comes from the fact that, the differential cross section presents a Rutherford-like small angle behavior with inverse powers of $(1-\cos \theta)$. Therefore, the angular dependence of the collision term would involve an infinite expansion of Legendre polynomials of the scattering angle, $\cos \theta$.

${ }^{26}$ In Ref. [91], bounds on the abundance of compact objects from gravitational lensing of type Ia supernovae are derived: compact objects represent less than $\sim 40 \%$ of the total matter content in the universe, at $95 \%$ confidence-level, thus excluding a scenario of all dark matter made up by primordial black holes. Reference [92] criticizes some aspects of Ref. [91] and shows that all-PBH dark matter scenario in the LIGO band is compatible with SN lensing constraints. The criticism is then addressed in the published version of [91], showing the validity of the constraints previously derived. However, in the case of a monochromatic mass distribution and no-clustering assumption EROS bounds [93] hold and disfavor a value $q=1$ in this mass range.
}

and/or peaked at $M \ll M_{\odot}$. Observational bounds on the relative abundance of $\mathrm{PBH}$ for $M \ll M_{\odot}$ comes mainly from the EROS microlensing survey [93] and from the cosmic microwave background temperature anisotropies and spectral distortions [94]. However, these bounds have recently been reinvestigated: EROS limits can easily be evaded [95-97], e.g., when considering more realistic dark matter distributions in the galaxy or if most $\mathrm{PBH}$ are regrouped in microclusters. On the other hand, Planck limits on PBH abundances have been shown to be very sensitive to the $\mathrm{PBH}$ velocity with respect to baryons, while there is no relevant constraint from CMB spectral distortions $[98,99]$. This reopened the low-mass window for PBH as dark matter candidate.

The generation of polarization is considerably enhanced at lower frequencies: in the LISA band the suppression of polarization with respect to anisotropies if $\sim 10^{-9}$ for both an astrophysical and cosmological background while for PTA the effect is enhanced by a factor 100 and the suppression of polarization with respect to anisotropies is of order $10^{-7}$. The estimate in the LISA band is derived assuming that the average fraction of stars in white dwarfs at extragalactic scales is the local one. The results for PTA can be further enhanced in a scenario in which PBH have a mass distribution peaked at $M \ll M_{\odot}$. Just to have a term of comparison, for the $\mathrm{CMB}$, the amount of polarization generated is suppressed by a factor $\sim 10^{-2}$ with respect to temperature fluctuations.

Our results are particularly interesting for a cosmological GW background. For this case the primary polarization vanishes and an upper bound on polarization can set upper bounds on the abundance of unresolved objects in the universe. For example, setting an upper bound on polarization in the LISA band, would set a bound on the extragalactic value of $f_{\text {wd }}$, see Eq. (4.46), which we have set equal to its local value in our estimates. Analogously, observing in the PTA band, an enhancement of polarization may provide information on the PHB mass distribution and abundance in the window $M \ll M_{\odot}$.

We emphasize that the aim of the estimates in Sec. IV is to get an idea of the size of the effect and of the kind of information that could in principle be extracted if polarization is measured or if stringent upper bounds are obtained. In particular, in Sec. IV we have introduced the simplifying hypothesis that the distribution of targets is isotropic. As explained, this assumption is consistent as long as we are interested in obtaining an estimate of the average amount of polarization, integrated over directions at the detector position. A direction dependent visibility function can be introduced as in Sec. III C and the more refined setting of Secs. II and III can be used to derive more precise results. In particular, since astrophysical objects acting as targets are embedded in galaxies which in turn belong to clusters etc., we expect polarization to have a pronounced directional dependence. Therefore, even if the 
averaged degree of polarization is very small, it may be much stronger and possibly detectable in the direction of compact structures or when correlated with matter overdensities. Detailed evaluations of experimental possibilities are left for future work. Moreover, as explained in Sec. III, GW polarization is a stochastic quantity which can be characterized statistically in terms of its angular power spectrum and its cross-correlation with intensity, in full analogy with the CMB polarization. From an observational point of view, methods are already available to reconstruct a polarization map of the sky, see e.g., the review [47]. The algorithm proposed in [100] to reconstruct a sky map of intensity can also be generalized to polarization. Comparing a sky map of polarization with theoretical predictions can be extremely interesting to reconstruct a chart of the invisible universe. For example, measuring an overproduction of polarization in a given direction would be an indication of the presence of a dense structure, e.g., a cluster (resolved or unresolved) in that direction.

\section{ACKNOWLEDGMENTS}

We thank Cyril Pitrou and Jean-Philippe Uzan for valuable comments and discussions during various stages of this work and for their help in the derivation of the emissivity function in the Boltzman approach. We are grateful to Macarena Lagos and Bernard Whiting for very useful discussions. We thank Michele Maggiore for an inspiring conversation at the early stage of this work. Finally, we thank Irina Dvorkin for comments on the astrophysical part of our study, Pierre Fleury and Davide Racco for discussions and references on primordial black holes and Enrico Barausse, Laura Bernard, Luc Blanchet and Guillaume Faye for useful references on GW diffusion. G. C. acknowledges financial support from ERC Grant No. 693024 and Beecroft Trust, R. D. acknowledges funding from the Swiss National Science Foundation. P. G. F acknowledges support from Beecroft Trust, ERC Grant 1379 No. 693024 and STFC.

\section{APPENDIX A: FORMAL RESULTS ON POLARIZATION TENSORS}

We work in transverse traceless gauge (TT). Using a plane wave expansion we can write a generic GW in TT gauge as

$$
h_{i j}^{T T}(t, \mathbf{x})=\int d f \int d^{2} \mathbf{n} \tilde{h}_{i j}(f, \mathbf{n}) e^{-2 \pi i f(t-\mathbf{n} \cdot \mathbf{x} / c)},
$$

and the Fourier components of the metric perturbation $h_{i j}(t, \mathbf{x})$ can be expanded in terms of either the linear polarization basis tensors

$$
\tilde{h}_{i j}(f, \mathbf{n})=\tilde{h}_{+}(f, \mathbf{n}) e_{i j}^{+}(\mathbf{n})+\tilde{h}_{\times}(f, \mathbf{n}) e_{i j}^{\times}(\mathbf{n}),
$$

or the circular polarization basis tensors

$$
\tilde{h}_{i j}(f, \mathbf{n})=\tilde{h}_{R}(f, \mathbf{n}) e_{i j}^{R}(\mathbf{n})+\tilde{h}_{L}(f, \mathbf{n}) e_{i j}^{L}(\mathbf{n}),
$$

where the circular polarization basis is defined in Eqs. (2.22) and (2.23). The expansion coefficients $\tilde{h}_{R}$, $\tilde{h}_{L}$ are related to $\tilde{h}_{+}, \tilde{h}_{\times}$through

$$
\begin{aligned}
& \tilde{h}_{R}=\frac{1}{\sqrt{2}}\left(h_{+}-i h_{\times}\right), \\
& \tilde{h}_{L}=\frac{1}{\sqrt{2}}\left(h_{+}+i h_{\times}\right) .
\end{aligned}
$$

Summarizing, we write

$h_{i j}^{T T}(t, \mathbf{x})=\sum_{A} \int d f \int d^{2} \mathbf{n} \tilde{h}_{A}(f, \mathbf{n}) e_{i j}^{A}(\mathbf{n}) e^{-2 \pi i f(t-\mathbf{n} \cdot \mathbf{x} / c)}$,

where the sum is over the two tensors of the polarization basis, i.e., $A=(+, \times)$ if we are using the linear polarization basis and $A=(R, L)$ if we are using the circular polarization basis.

\section{General properties of polarization tensors}

The polarization tensors $e_{i j}^{A}(\mathbf{n})$ satisfy

$$
e_{i j}^{A}(\mathbf{n}) e^{B i j *}(\mathbf{n})=2 \delta^{A B} .
$$

Under a rotation of an angle $\psi$ in the plane orthogonal to the n direction, the polarization basis $\left(e_{i j}^{+}, e_{i j}^{\times}\right)$transforms as

$$
\begin{gathered}
e_{i j}^{+}(\mathbf{n}, \psi)=\cos 2 \psi e_{i j}^{+}(\mathbf{n})+\sin 2 \psi e_{i j}^{\times}(\mathbf{n}), \\
e_{i j}^{\times}(\mathbf{n}, \psi)=-\sin 2 \psi e_{i j}^{+}(\mathbf{n})+\cos 2 \psi e_{i j}^{\times}(\mathbf{n}) .
\end{gathered}
$$

while the basis $\left(e_{i j}^{R}, e_{i j}^{L}\right)$ transforms as

$$
\begin{aligned}
& e_{i j}^{R}(\mathbf{n}, \psi)=e^{-i 2 \psi} e_{i j}^{R}(\mathbf{n}), \\
& e_{i j}^{L}(\mathbf{n}, \psi)=e^{i 2 \psi} e_{i j}^{L}(\mathbf{n}) .
\end{aligned}
$$

The tensors $e_{i j}^{R}$ and $e_{i j}^{L}$ corresponds to right and left circularly polarized waves.

Let us construct the basis of polarization tensors $\left(e_{i j}^{\times}(\mathbf{n}), e_{i j}^{+}(\mathbf{n})\right)$. We can choose an orthonormal basis in the plane normal to the direction of propagation $\mathbf{n}$, i.e., $\mathbf{u}(\mathbf{n})$ and $\mathbf{v}(\mathbf{n})$. We define

$$
\begin{aligned}
& e_{i j}^{+}(\mathbf{n})=u_{i}(\mathbf{n}) u_{j}(\mathbf{n})-v_{i}(\mathbf{n}) v_{j}(\mathbf{n}), \\
& e_{i j}^{\times}(\mathbf{n})=u_{i}(\mathbf{n}) v_{j}(\mathbf{n})+v_{i}(\mathbf{n}) u_{j}(\mathbf{n}) .
\end{aligned}
$$


Using the fact that $\mathbf{v}$ and $\mathbf{u}$ are orthonomal, one can verify that Eq. (A7) is satisfied. We compute the contraction between polarization vectors relative to different directions of propagation. We use the shortcut notation $e_{i j}^{A}\left(\mathbf{n}^{\prime}\right)=e_{i j}^{\prime A}$. We have

$$
\begin{aligned}
& e_{i j}^{\prime+} e^{i j+}=\left(\mathbf{u} \cdot \mathbf{u}^{\prime}\right)^{2}+\left(\mathbf{v} \cdot \mathbf{v}^{\prime}\right)^{2}-\left(\mathbf{v} \cdot \mathbf{u}^{\prime}\right)^{2}-\left(\mathbf{u} \cdot \mathbf{v}^{\prime}\right)^{2}, \\
& e_{i j}^{\prime \times} e^{i j \times}=2\left(\mathbf{u} \cdot \mathbf{u}^{\prime}\right)\left(\mathbf{v} \cdot \mathbf{v}^{\prime}\right)+2\left(\mathbf{v} \cdot \mathbf{u}^{\prime}\right)\left(\mathbf{u} \cdot \mathbf{v}^{\prime}\right), \\
& e_{i j}^{\prime \times} e^{i j+}=2\left(\mathbf{u} \cdot \mathbf{u}^{\prime}\right)\left(\mathbf{u} \cdot \mathbf{v}^{\prime}\right)-2\left(\mathbf{v} \cdot \mathbf{v}^{\prime}\right)\left(\mathbf{v} \cdot \mathbf{u}^{\prime}\right), \\
& e_{i j}^{\prime+} e^{i j \times}=2\left(\mathbf{u} \cdot \mathbf{u}^{\prime}\right)\left(\mathbf{v} \cdot \mathbf{u}^{\prime}\right)-2\left(\mathbf{v} \cdot \mathbf{v}^{\prime}\right)\left(\mathbf{u} \cdot \mathbf{v}^{\prime}\right) .
\end{aligned}
$$

One can verify that ${ }^{27}$

$\sum_{A B}\left|e_{i j}^{A}\left(\mathbf{n}^{\prime}\right) e^{i j B}(\mathbf{n})\right|^{2}=1+\left(\mathbf{n} \cdot \mathbf{n}^{\prime}\right)^{4}+6\left(\mathbf{n} \cdot \mathbf{n}^{\prime}\right)^{2}$.

\section{APPENDIX B: BOLTZMAN APPROACH}

We propose an alternative derivation of the results of $[55,56]$ using a Boltzmann approach. ${ }^{28}$ We will see that the geometric optics approximation used in [55,56] corresponds to the zero-collision hypothesis in the Boltzmann treatment. We also derive result for the anisotropies of a cosmological background of radiation.

We introduce a distribution function of gravitons $f\left(x^{\mu}, k^{\mu}\right)$, satisfying the following Boltzman equation

$$
\mathcal{L}[f]=\mathcal{E}[\lambda]+\mathcal{C}[f]
$$

where $\mathcal{L} \equiv d / d \eta$ is the Liouville operator and $\mathcal{E}, \mathcal{C}$ and emissivity term and collision term respectively. We denote by $\lambda$ the affine parameter along the geodesic with tangent vector $k^{\mu}$ so that $d \eta / d \lambda=k^{0}=\omega$. In this Appendix we denote the frequency by $\omega$, to avoid confusion with the distribution function.

We introduce two reference frames, a cosmological reference frame and a reference frame tied to baryons (and galaxies under the assumption that the galaxy velocity is unbiased). We then have

$$
e^{\mu}{ }_{a}=\left(u^{\mu}, e^{\mu}{ }_{i}\right), \quad\left(e^{\mu}{ }_{a}\right)_{\mathrm{G}}=\left(u^{\mu}, e^{\mu}{ }_{i}\right)_{\mathrm{G}},
$$

\footnotetext{
${ }^{27}$ One way to verify this result is to pick up a specific choice for $\mathbf{n}, \mathbf{n}^{\prime}$ and for the vectors of the polarization basis, expressing the final result in a coordinate independent way. For example we can choose $\mathbf{n}=(\sin \theta, \cos \theta, 0), \mathbf{n}^{\prime}=(0,1,0)$ and $\mathbf{u}^{\prime}=\mathbf{e}_{x}, \mathbf{v}^{\prime}=\mathbf{e}_{z}$ and $\mathbf{u}=(\cos \theta,-\sin \theta, 0), \mathbf{v}=(0,0,1)$. One finds $e_{i j}^{\prime+} e^{i j+}=$ $1+\cos \theta^{2}, e_{i j}^{\prime \times} e^{i j \times}=2 \cos \theta$ and the mixed terms are vanishing. We then simply insert $\cos \theta=\mathbf{n} \cdot \mathbf{n}^{\prime}$.

${ }^{28}$ See also [101] for a first attempt to derive anisotropies from a Boltzmann approach.
}

which are related by a boost of velocity $\mathbf{v}$ as

$$
e^{\mu}{ }_{a}=\Lambda_{\nu}^{\mu}\left(e^{\nu}{ }_{a}\right)_{\mathrm{G}},
$$

see Appendix G of [102] for details on how physical quantities transform under this boost. If we have a graviton with 4-momentum $\left(k^{\mu}\right)=\omega\left(1, n^{i}\right)$, its energy and direction measured in the two frames are related as

$$
\begin{gathered}
\omega_{\mathrm{G}}=-\left(u_{\mu} k^{\mu}\right)_{\mathrm{G}}=\omega(1-\mathbf{n} \cdot \mathbf{v}), \\
n_{\mathrm{G}}^{i}=(1+\mathbf{n} \cdot \mathbf{v}) n^{i},
\end{gathered}
$$

to first order there is no aberration. The distribution function does not transform and one has

$$
f(\eta, \mathbf{x}, \omega, \mathbf{n})=f_{\mathrm{G}}\left(\eta, \mathbf{x}, \omega_{\mathrm{G}}, \mathbf{n}_{\mathrm{G}}\right) .
$$

From now on we therefore omit the subscript ${ }_{\mathrm{G}}$ on the distribution function. We define (in the frame of the comoving observer) $)^{29}$

$$
\Omega_{\mathrm{GW}}\left(\eta, \mathbf{x}, \mathbf{n}_{\mathrm{G}}\right) \equiv \frac{1}{\rho_{c}} \int d \omega_{\mathrm{G}} \omega_{\mathrm{G}}^{3} f\left(\eta, \mathbf{x}, \omega_{\mathrm{G}}, \mathbf{n}_{\mathrm{G}}\right) .
$$

The Liouville operator can be written as

$$
\mathcal{L}[\lambda]=\frac{d \eta}{d \lambda}\left[\frac{\partial f}{\partial \eta}+n^{i} \partial_{i} f+\frac{\partial f}{\partial \log \omega_{\mathrm{G}}} \frac{\partial \log \omega_{\mathrm{G}}}{\partial \eta}\right] .
$$

We observe that since we will be interested in quantities up to first order in perturbations, we can neglect all aberration effects in Eq. (B8), setting $\mathbf{n}=\mathbf{n}_{\mathrm{G}}$. Furthermore, we have assumed that $f$ has no intrinsic direction dependence so that the latter only enters via the dependence of $\omega_{G}$ on $\mathbf{n}$ via

$$
\frac{d \log \omega_{\mathrm{G}}}{d \eta}=\frac{d \log \omega}{d \eta}-n^{i} \frac{d v^{i}}{d \eta} .
$$

\section{Astrophysical background}

We neglect the collision term in Eq. (B1). We then have

$$
\frac{d f}{d \tau}=\frac{d \lambda}{d \tau} \mathcal{E}[\lambda]
$$

where $\tau$ is the proper time of the observer. We recall that $\mathcal{E}[\lambda]=$ (number gravitons produced) $/($ units of $\lambda)$. It follows that the quantity on the right-hand side of (B10) is proportional to the number of gravitons produced per units of $\tau$. We observe that

\footnotetext{
${ }^{29}$ For practical purposes, we are absorbing a factor $1 /(4 \pi)$ in the definition of $f$. To make contact with standard conventions, we will replace $\Omega_{\mathrm{GW}} \rightarrow 4 \pi \Omega_{\mathrm{GW}}$ in the final result.
} 


$$
\frac{d \rho_{\mathrm{GW}}}{d \tau}=\int d \omega_{\mathrm{G}} \omega_{\mathrm{G}}^{3} \frac{d f}{d \tau}=\int d \omega_{\mathrm{G}} \omega_{\mathrm{G}}^{3} \frac{d \lambda}{d \tau} \mathcal{E}[\lambda]
$$

The explicit form of the emissivity function depends on the physical situation being considered. Comparing with the astrophysical model of [55] we find

$$
\mathcal{E}[\lambda]=\left(\frac{d \tau}{d \lambda}\right) \mathcal{L}_{\mathrm{G}}\left(\vartheta_{\mathrm{G}}, \omega_{\mathrm{G}}\right) \frac{n_{\mathrm{G}}}{\omega_{\mathrm{G}}^{3}},
$$

where $\vartheta_{\mathrm{G}}$ are a set of parameters effectively describing a given galaxy (mass, metallicity, etc.). It follows that Eq. (B1) can be rewritten as

$$
\frac{d f}{d \eta}=\left(\frac{d \tau}{d \eta}\right) \mathcal{L}_{\mathrm{G}}\left(\vartheta_{\mathrm{G}}, \omega_{\mathrm{G}}\right) \frac{n_{\mathrm{G}}}{\omega_{\mathrm{G}}^{3}} .
$$

The relation between proper time and conformal time [55] is

$$
\frac{d \tau}{d \eta}=\sqrt{p^{\mu} p^{\nu} g_{\mu \nu}} \frac{d \lambda}{d \eta}=a[1+\psi-\mathbf{v} \cdot \mathbf{n}]
$$

where $p^{\mu}$ is the spatial projection of the graviton 4-vector.

We can now solve Eq. (B13) replacing (B14) and (B8), and then integrating over energy to find an equation for $\Omega_{\mathrm{GW}}$ The equation for the background becomes

$$
\bar{\Omega}_{\mathrm{GW}}\left(\eta_{0}\right)=\int_{0}^{\eta_{0}} d \eta a(\eta)^{5} \bar{n}_{\mathrm{G}}(\eta) \int d \eta_{\mathrm{G}} L_{\mathrm{G}}\left(\vartheta_{\mathrm{G}}\right)
$$

where we have introduced the integrated luminosity

$$
L_{\mathrm{G}}\left(\vartheta_{\mathrm{G}}\right)=\int d \omega_{\mathrm{G}} \mathcal{L}_{\mathrm{G}}\left(\omega_{\mathrm{G}}, \vartheta_{\mathrm{G}}\right)
$$

The equation for the perturbed quantity $\delta \Omega_{\mathrm{GW}}$ can be written as

$$
\begin{aligned}
\partial_{\eta} \delta \Omega_{\mathrm{GW}}+n^{i} \partial_{i} \delta \Omega_{\mathrm{GW}}+4 \mathcal{H} \delta \Omega_{\mathrm{GW}} \\
=-4\left[\frac{d}{d \eta}(\phi+\mathbf{n} \cdot \mathbf{v})-\dot{\psi}-\dot{\phi}\right] \bar{\Omega}_{\mathrm{GW}} \\
\quad+\bar{n}_{\mathrm{G}} a\left(\psi-\mathbf{v} \cdot \mathbf{n}+\delta_{\mathrm{G}}\right) \int d \vartheta_{\mathrm{G}} L_{\mathrm{G}} .
\end{aligned}
$$

This equation can be solved by writing the left-hand side as

$$
(\text { l.h.s. })=a^{-4} \frac{d}{d \eta}\left(a^{4} \delta \Omega_{\mathrm{GW}}\right)
$$

and after standard manipulations and replacing $\Omega_{\mathrm{GW}} \rightarrow$ $4 \pi \Omega_{\mathrm{GW}}$, we find

$$
\delta \Omega_{\mathrm{GW}}(\mathbf{e})=\frac{1}{4 \pi \rho_{c}} \int_{0}^{\eta_{0}} d \eta a^{5} \bar{n}_{\mathrm{G}}(\eta)\left[\delta_{\mathrm{G}}+4 \phi+\psi-3 \mathbf{e} \cdot \mathbf{v}+4 \int_{\eta}^{\eta_{0}} d \eta^{\prime}(\dot{\phi}+\dot{\psi})\right] \int d \vartheta_{\mathrm{G}} L_{\mathrm{G}}\left(\vartheta_{\mathrm{G}}\right)
$$

The corresponding result per logarithmic frequency can be obtained using Eq. (B16), recalling that $\omega_{\mathrm{G}}=\left(1+z_{\mathrm{G}}\right) \omega$ and taking into account redshift perturbations is

$$
\Omega_{\mathrm{GW}}(\omega, \mathbf{e})=\frac{\omega}{4 \pi \rho_{c}} \int_{0}^{\eta_{0}} d \eta a^{4} \bar{n}_{\mathrm{G}}(\eta)\left[1+\delta_{\mathrm{G}}+4 \psi-2 \mathbf{e} \cdot \nabla v+3 \int_{\eta}^{\eta_{0}} d \eta^{\prime}(\dot{\psi}+\dot{\phi})\right] \int d \vartheta_{\mathrm{G}} \mathcal{L}_{\mathrm{G}}\left(\omega_{\mathrm{G}}, \vartheta_{\mathrm{G}}\right),
$$

which agrees with the result given in Eq. (67) of [55].

\section{A cosmological background}

For a GW background of cosmological origin, we solve (B1) with stochastic initial conditions for the various fields (and no emissivity). One has

$$
\bar{\Omega}_{\mathrm{GW}}\left(\eta_{0}\right)=\frac{a\left(\eta_{i}\right)^{4}}{a\left(\eta_{0}\right)^{4}} \bar{\Omega}_{\mathrm{GW}}\left(\eta_{i}\right)
$$

and

$$
\begin{aligned}
\frac{\delta \Omega_{\mathrm{GW}}\left(\eta_{0}, \mathbf{e}\right)}{\bar{\Omega}_{\mathrm{GW}}\left(\eta_{0}\right)}= & \frac{\delta \Omega_{\mathrm{GW}}\left(\eta_{i}, \mathbf{e}\right)}{\bar{\Omega}_{\mathrm{GW}}\left(\eta_{i}\right)}-4\left[\phi_{0}-\phi_{i}-\mathbf{e} \cdot \mathbf{v}_{0}+\mathbf{e} \cdot \mathbf{v}_{i}\right] \\
& +4 \int_{\eta_{i}}^{\eta_{0}} d \eta(\dot{\phi}+\dot{\psi}),
\end{aligned}
$$

where $\eta_{i}$ is the initial time at which the background is produced. The result per units of logarithmic frequency is simply

$$
\begin{aligned}
\frac{\delta \Omega_{G W}\left(\eta_{0}, \mathbf{e}, \omega\right)}{\bar{\Omega}_{G W}\left(\eta_{0}, \omega\right)}= & \frac{\delta \Omega_{G W}\left(\eta_{i}, \mathbf{e}, \omega\right)}{\bar{\Omega}_{G W}\left(\eta_{i}, \omega\right)} \\
& -4\left[\phi_{0}-\phi_{i}-\mathbf{e} \cdot \mathbf{v}_{0}+\mathbf{e} \cdot \mathbf{v}_{i}\right] \\
& +4 \int_{\eta_{i}}^{\eta_{0}} d \eta(\dot{\phi}+\dot{\psi}) .
\end{aligned}
$$

\section{APPENDIX C: THE AMPLITUDE OF FLUCTUATIONS}

\section{Astrophysical background}

An order of magnitude estimate of the amplitude of the anisotropies of the astrophysical background with 
respect to the isotropic component can be obtained by computing

$$
\begin{aligned}
\sigma_{\mathrm{GW}}^{2}(f) & \equiv \frac{\left\langle\delta \Omega_{\mathrm{GW}}(\mathbf{e}, f) \delta \Omega_{\mathrm{GW}}(\mathbf{e}, f)\right\rangle}{\bar{\Omega}_{\mathrm{GW}}^{2}(f)} \\
& =\frac{1}{\bar{\Omega}_{\mathrm{GW}}^{2}(f)} \sum_{\ell} \frac{2 \ell+1}{2 \pi} C_{\ell}(f) .
\end{aligned}
$$

For the second equality we have used that the correlation function.

$$
C(f, \theta)=\left\langle\delta \Omega_{\mathrm{GW}}\left(f, \mathbf{e}_{1}\right) \delta \Omega_{\mathrm{GW}}\left(f, \mathbf{e}_{2}\right)\right\rangle,
$$

with $\mathbf{e}_{1} \cdot \mathbf{e}_{2}=\cos \theta$ can be expanded in Legendre polynomials as

$$
C(f, \theta) \equiv \sum_{\ell} \frac{2 \ell+1}{2 \pi} C_{\ell}(f) P_{\ell}\left(\mathbf{e}_{1} \cdot \mathbf{e}_{2}\right)
$$

The angular power spectrum has the following expression, see [55]

$$
C_{\ell}(f)=\frac{2}{\pi} \int \mathrm{d} k k^{2}\left|\delta \Omega_{\ell}(k, f)\right|^{2}
$$

Here $k$ is the Fourier variable and $\delta \Omega_{\ell}(k, f)$ is the $\ell$-mode of the Fourier transform of $\delta \Omega_{\ell}(\mathbf{x}, f)$. On large scales it is simply given by [55]

$$
\begin{aligned}
\delta \Omega_{\ell}(k, f)= & \frac{f}{4 \pi \rho_{c}} \int_{0}^{\eta_{\mathrm{O}}} \mathrm{d} \eta a^{5}(\eta) \bar{n}_{\mathrm{G}}(\eta) b \delta_{k}(\eta) j_{\ell}(k \Delta \eta) \\
& \times \int d \vartheta_{\mathrm{G}} \mathcal{L}_{\mathrm{G}}\left(f_{\mathrm{G}}, \vartheta_{\mathrm{G}}\right),
\end{aligned}
$$

where $b$ is the (scale-independent) bias and $\delta_{k}$ the matter over density (in Fourier space). We work under the following hypotheses:

(1) all galaxies have the same integrated luminosity (i.e., $\mathcal{L}_{\mathrm{G}}(f)$ does not depend on $\vartheta_{\mathrm{G}}$ );

(2) the luminosity (integrated) does not depend on time, i.e., the luminosity per units of frequency scales with a simple redshift factor

$$
\begin{aligned}
\mathcal{L}_{\mathrm{G}}\left(f_{\mathrm{G}}, \vartheta_{\mathrm{G}}\right) & =\mathcal{L}_{\mathrm{G}}\left(f, \vartheta_{\mathrm{G}}\right) \frac{d f}{d f_{\mathrm{G}}} \\
& =\left(1+z_{\mathrm{G}}\right)^{-1} \mathcal{L}_{\mathrm{G}}\left(f, \vartheta_{\mathrm{G}}\right)
\end{aligned}
$$

(3) the universe is matter dominated, $a(\eta)=\left(\eta / \eta_{\mathrm{O}}\right)^{2}$; (4) $\delta_{m}=\delta_{\mathrm{CDM}}$ (we neglect baryons).

We focus on subhorizon modes in matter domination, i.e., on $k \gg k_{\text {eq }}$, for which

$$
\delta_{\mathrm{k}}(\eta)=\delta_{\mathrm{k}}\left(\frac{\eta}{\eta_{\mathrm{eq}}}\right)^{2}
$$

with

$$
\delta_{\mathrm{k}}=\frac{9}{10} \Phi_{\mathrm{k}}^{P}\left[-1+6 \log \left(k \eta_{\mathrm{eq}}\right)\right],
$$

with the primordial power spectrum given by

$$
\Phi_{k}^{P}=\pi \sqrt{2} \frac{2}{3} k^{-3 / 2} \frac{A_{\mathrm{S}}^{1 / 2}}{\left[1+\frac{4}{15} R_{\nu}\right]}\left(\frac{k}{k_{\mathrm{ref}}}\right)^{\left(n_{s}-1\right) / 2},
$$

where $R_{\nu}$ is the fraction of neutrinos in radiation $R_{\nu} \equiv$ $\Omega_{\nu} /\left(\Omega_{\nu}+\Omega_{\gamma}\right)=\alpha /(1+\alpha)$ with $\alpha=N_{\text {eff }} 7 / 8(4 / 11)^{4 / 3}$ and $N_{\text {eff }}=3.046$ is the effective number of neutrino species, see e.g., [103]. ${ }^{30}$ We use

$$
\bar{n}_{\mathrm{G}}(\eta)=n_{\mathrm{G}, \mathrm{O}}\left(\frac{a_{\mathrm{O}}}{a}\right)^{3}=n_{\mathrm{G}, \mathrm{O}}\left(\frac{\eta_{\mathrm{O}}}{\eta}\right)^{6} .
$$

and we assume the bias to be scale-independent and to scale as $\propto \sqrt{1+z}[105,106]:$

$$
b \sim b_{\mathrm{O}} \sqrt{1+z}=b_{\mathrm{O}}\left(\frac{\eta_{\mathrm{O}}}{\eta}\right),
$$

with $b_{\mathrm{O}} \sim 1$. Putting all these ingredients together and making use of the following asymptotic behavior of the spherical Bessel function

$$
j_{\ell}(x) \sim \sqrt{\frac{\pi}{2 \ell+1}} \delta\left(\ell+\frac{1}{2}-x\right)+\mathcal{O}\left(1 / \ell^{2}\right),
$$

Eq. (C5) simplifies to

$\delta \hat{\Omega}_{\ell}(k, f)=\frac{f}{4 \pi \rho_{c}} \mathcal{L}_{\mathrm{G}}(f) b_{\mathrm{O}} n_{\mathrm{G}, \mathrm{O}} \frac{1}{k}\left(\frac{\eta_{\mathrm{O}}}{\eta_{\mathrm{eq}}}\right)^{2}\left(\frac{\bar{\eta}}{\eta_{\mathrm{O}}}\right)^{7} \sqrt{\frac{\pi}{2 \ell+1}} \delta_{k}$,

with

$$
\bar{\eta}=\eta_{\mathrm{O}}-\left(\ell+\frac{1}{2}\right) \frac{1}{k}
$$

which can be approximated to $\bar{\eta}=\eta_{\mathrm{O}}$ for large enough angular scales. Then

$$
\begin{aligned}
C_{\ell}(f)= & \left(\ell+\frac{1}{2}\right)^{-1}\left[\frac{f}{4 \pi \rho_{c}} \mathcal{L}_{\mathrm{G}}(f) b_{\mathrm{O}} n_{\mathrm{G}, \mathrm{O}}\right]^{2}\left(\frac{\eta_{\mathrm{O}}}{\eta_{\mathrm{eq}}}\right)^{4} \\
& \times \int_{\mathrm{k}_{\mathrm{eq}}} d \mathrm{k}\left|\delta_{\mathrm{k}}\right|^{2}
\end{aligned}
$$

\footnotetext{
${ }^{30}$ All values for the cosmological parameters are those of [104], explicitly $k_{\text {ref }}=0.002 \mathrm{Mpc}, A_{\mathrm{S}}=2.1986 \times 10^{-9}, n_{s}=0.9652$.
} 
with $^{31}$

$$
\begin{aligned}
\int_{\mathrm{k}_{\mathrm{eq}}} d \mathrm{k}\left|\delta_{\mathrm{k}}\right|^{2} \simeq & \pi^{2} \frac{8}{9}\left(\frac{9}{10}\right)^{2} \frac{1}{3-n_{s}}\left[\frac{A_{\mathrm{S}}^{1 / 2}}{1+\frac{4}{15} R_{\nu}}\right]^{2} \\
& \times\left(\frac{k_{\mathrm{ref}}}{k_{\mathrm{eq}}}\right)^{1-n_{s}}\left(\frac{\eta_{\mathrm{eq}}}{\eta_{\mathrm{O}}}\right)^{2} \eta_{\mathrm{O}}^{2} .
\end{aligned}
$$

While for the isotropic component we have

$$
\bar{\Omega}_{\mathrm{GW}}^{2}(f) \simeq \frac{1}{25}\left[\frac{f}{4 \pi \rho_{c}} \mathcal{L}_{\mathrm{G}}(f) n_{\mathrm{G}, \mathrm{O}}\right]^{2},
$$

Going back to Eq. (4.17) and using Eqs. (C15)-(C17), we find

$$
\sigma_{\mathrm{GW}}^{2} \simeq 18 \pi b_{\mathrm{O}}^{2}\left[\frac{A_{\mathrm{S}}^{1 / 2}}{1+\frac{4}{15} R_{\nu}}\right]^{2}\left(\frac{k_{\mathrm{ref}}}{k_{\mathrm{eq}}}\right)^{1-n_{\mathrm{s}}}\left(\frac{\eta_{\mathrm{O}}}{\eta_{\mathrm{eq}}}\right)^{2} \sim 10^{-4} .
$$

We can conclude that the relative amplitude of fluctuations with respect to the isotropic component, for a generic astrophysical background is of order

$$
\frac{\delta \Omega_{\mathrm{GW}}}{\bar{\Omega}_{\mathrm{GW}}} \sim \sigma_{\mathrm{GW}} \approx 10^{-2} .
$$

This result applies to any component of the astrophysical background as long as the luminosity function $\mathcal{L}_{\mathrm{G}}$ depends

\footnotetext{
${ }^{31}$ We neglect the dependence on the logarithm in Eq. (C7).
}

on time only through the redshifted frequency [see Eq. (C6)], which is the case e.g., for a background of mergers in the LIGO band. The estimate can be refined by assuming for each contribution to the background (black hole mergers, supernovae, ...) a specific frequency dependence of the luminosity function. Equation (C19) is in agreement with the numerical results obtained in [57] for the case of a background of black hole mergers.

Note that (C19) is an estimate of the amount of anisotropy today, at $\eta=\eta_{0}$. The analogous quantity at a different time $\eta_{1}<\eta_{0}$, can be worked out in a similar way: in the definition of the energy density (B20), the integral over time runs up to $\eta_{1}$ and Eq. (C13) has to be evaluated at $\bar{\eta}_{1}=\eta_{1}-(\ell+1 / 2) / k$. For $\eta_{1} \gg \eta_{\text {eq }}$, one has $\eta_{1} / \eta_{0} \gg(\ell+1 / 2) /\left(\eta_{0} k_{\text {eq }}\right) \gg(\ell+1 / 2) /\left(\eta_{0} k\right)$, hence $\overline{\eta_{1}} / \eta_{0} \simeq \eta_{1} / \eta_{0}$ and the final result for the fluctuations is suppressed by a factor $\left(\eta_{1} / \eta_{0}\right)^{7}$ with respect to (C19). In Sec. IV we consider a simplified framework where most of astrophysical sources are located at redshift $z=1.5$. For this situation $\delta \Omega_{\mathrm{GW}} / \bar{\Omega}_{\mathrm{GW}}\left(\eta_{1}\right) \simeq\left(\eta_{1} / \eta_{0}\right)^{7} \delta \Omega_{\mathrm{GW}} / \bar{\Omega}_{\mathrm{GW}}\left(\eta_{0}\right) \simeq$ $\left(a_{0} / a_{1}\right)^{7 / 2} \delta \Omega_{\mathrm{GW}} / \bar{\Omega}_{\mathrm{GW}}\left(\eta_{0}\right) \simeq 10^{-3}$.

\section{Cosmological background}

The anisotropies of a cosmological GW background are a tracer of the temperature anisotropies of the CMB. We therefore expect $\delta \Omega_{\mathrm{GW}} / \bar{\Omega}_{\mathrm{GW}}\left(\eta_{0}\right) \simeq 10^{-5}$. If we consider $\eta_{1}<\eta_{0}$, the result changes slightly due to the change of the integration bounds of the integrated Sachs-Wolfe term in Eq. (B23). As a first approximation, we neglect this correction, assuming that the value $10^{-5}$ stays the same for any redshift.
[1] A. A. Penzias and R. W. Wilson, A measurement of excess antenna temperature at 4080-Mc/s, Astrophys. J. 142, 419 (1965).

[2] M. G. Hauser and E. Dwek, The cosmic infrared background: Measurements and implications, Annu. Rev. Astron. Astrophys. 39, 249 (2001).

[3] R. B. Partridge and P. J. E. Peebles, Are Young Galaxies visible? II. The integrated background, Astrophys. J. 148, 377 (1967).

[4] T. Shanks, I. Georgantopoulos, G. C. Stewart, K. A. Pounds, B. J. Boyle, and R. E. Griffiths, The origin of the cosmic X-ray background, Nature (London) 353, 315 (1991).

[5] S. Hannestad, Primordial neutrinos, Annu. Rev. Nucl. Part. Sci. 56, 137 (2006).

[6] P. Peter and J.-P. Uzan, Primordial Cosmology, Oxford Graduate Texts (Oxford University Press, Oxford, 2005).

[7] J. F. Dufaux, A. Bergman, G. N. Felder, L. Kofman, and J.-P. Uzan, Theory and numerics of gravitational waves from preheating after inflation, Phys. Rev. D 76, 123517 (2007).
[8] A. Vilenkin, Gravitational radiation from cosmic strings, Phys. Lett. 107B, 47 (1981).

[9] C. J. Hogan and M. J. Rees, Gravitational interactions of cosmic strings, Nature (London) 311, 109 (1984).

[10] T. Vachaspati and A. Vilenkin, Gravitational radiation from cosmic strings, Phys. Rev. D 31, 3052 (1985).

[11] R. R. Caldwell and B. Allen, Cosmological constraints on cosmic string gravitational radiation, Phys. Rev. D 45, 3447 (1992).

[12] S. Kuroyanagi, K. Takahashi, N. Yonemaru, and H. Kumamoto, Anisotropies in the gravitational wave background as a probe of the cosmic string network, Phys. Rev. D 95, 043531 (2017).

[13] C. Caprini, R. Durrer, T. Konstandin, and G. Servant, General properties of the gravitational wave spectrum from phase transitions, Phys. Rev. D 79, 083519 (2009).

[14] C. Caprini et al., Science with the space-based interferometer eLISA. II: Gravitational waves from cosmological 
phase transitions, J. Cosmol. Astropart. Phys. 04 (2016) 001.

[15] C. Caprini and R. Durrer, Gravitational wave production: A strong constraint on primordial magnetic fields, Phys. Rev. D 65, 023517 (2001).

[16] P. Binetruy, A. Bohe, C. Caprini, and J.-F. Dufaux, Cosmological backgrounds of gravitational waves and eLISA/NGO: Phase transitions, cosmic strings and other sources, J. Cosmol. Astropart. Phys. 06 (2012) 027.

[17] C. Caprini and D. G. Figueroa, Cosmological backgrounds of gravitational waves, Classical Quantum Gravity 35, 163001 (2018).

[18] M. Maggiore, Gravitational wave experiments and early universe cosmology, Phys. Rep. 331, 283 (2000).

[19] A. Buonanno and B. S. Sathyaprakash, Sources of gravitational waves: Theory and observations, arXiv:1410 .7832 .

[20] B. P. Abbott et al. (Virgo and LIGO Scientific Collaborations), GW150914: Implications for the Stochastic Gravitational Wave Background from Binary Black Holes, Phys. Rev. Lett. 116, 131102 (2016).

[21] T. Regimbau, M. Evans, N. Christensen, E. Katsavounidis, B. Sathyaprakash, and S. Vitale, Digging Deeper: Observing Primordial Gravitational Waves below the Binary Black Hole Produced Stochastic Background, Phys. Rev. Lett. 118, 151105 (2017).

[22] V. Mandic, S. Bird, and I. Cholis, Stochastic GravitationalWave Background due to Primordial Binary Black Hole Mergers, Phys. Rev. Lett. 117, 201102 (2016).

[23] I. Dvorkin, J.-P. Uzan, E. Vangioni, and J. Silk, Synthetic model of the gravitational wave background from evolving binary compact objects, Phys. Rev. D 94, 103011 (2016).

[24] K. Nakazato, Y. Niino, and N. Sago, Gravitational-wave background from binary mergers and metallicity evolution of Galaxies, Astrophys. J. 832, 146 (2016).

[25] I. Dvorkin, E. Vangioni, J. Silk, J.-P. Uzan, and K. A. Olive, Metallicity-constrained merger rates of binary black holes and the stochastic gravitational wave background, Mon. Not. R. Astron. Soc. 461, 3877 (2016).

[26] E. F. D. Evangelista and J. C. N. Araujo, The gravitational wave background from coalescing compact binaries: A new method, Braz. J. Phys. 44, 824 (2014).

[27] L. Z. Kelley, L. Blecha, L. Hernquist, and A. Sesana, The gravitational wave background from massive black hole binaries in illustris: Spectral features and time to detection with pulsar timing arrays, Mon. Not. R. Astron. Soc. 471, 4508 (2017).

[28] M. Surace, K. D. Kokkotas, and P. Pnigouras, The stochastic background of gravitational waves due to the $f$-mode instability in neutron stars, Astron. Astrophys. 586, A86 (2016).

[29] D. Talukder, E. Thrane, S. Bose, and T. Regimbau, Measuring neutron-star ellipticity with measurements of the stochastic gravitational-wave background, Phys. Rev. D 89, 123008 (2014).

[30] P. D. Lasky, M. F. Bennett, and A. Melatos, Stochastic gravitational wave background from hydrodynamic turbulence in differentially rotating neutron stars, Phys. Rev. D 87, 063004 (2013).
[31] K. Crocker, T. Prestegard, V. Mandic, T. Regimbau, K. Olive, and E. Vangioni, A systematic study of the stochastic gravitational-wave background due to stellar core collapse, Phys. Rev. D 95, 063015 (2017).

[32] K. Crocker, V. Mandic, T. Regimbau, K. Belczynski, W. Gladysz, K. Olive, T. Prestegard, and E. Vangioni, Model of the stochastic gravitational-wave background due to core collapse to black holes, Phys. Rev. D 92, 063005 (2015).

[33] I. Kowalska, T. Bulik, and K. Belczynski, Gravitational wave background from population III binaries, Astron. Astrophys. 541, A120 (2012).

[34] B. P. Abbott et al. (Virgo and LIGO Scientific Collaborations), Observation of Gravitational Waves from a Binary Black Hole Merger, Phys. Rev. Lett. 116, 061102 (2016).

[35] B. P. Abbott et al. (LIGO Scientific and Virgo Collaborations), GWTC-1: A Gravitational-Wave Transient Catalog of Compact Binary Mergers Observed by LIGO and Virgo during the First and Second Observing Runs, Phys. Rev. Lett. 119, 161101 (2017).

[36] B. Abbott et al. (Virgo and LIGO Scientific Collaborations), GW170817: Observation of Gravitational Waves from a Binary Neutron Star Inspiral, Phys. Rev. Lett. 119, 161101 (2017).

[37] B. P. Abbott et al. (Virgo and LIGO Scientific Collaborations), GW170817: Implications for the Stochastic Gravitational-Wave Background from Compact Binary Coalescences, Phys. Rev. Lett. 120, 091101 (2018).

[38] B. Allen, The stochastic gravity wave background: Sources and detection, in Relativistic gravitation and gravitational radiation. Proceedings, School of Physics, Les Houches, France, 1995 (Cambridge Contemporary Astrophysics, Cambridge, 1996), pp. 373-417.

[39] T. L. Smith, E. Pierpaoli, and M. Kamionkowski, A New Cosmic Microwave Background Constraint to Primordial Gravitational Waves, Phys. Rev. Lett. 97, 021301 (2006).

[40] S. Henrot-Versille et al., Improved constraint on the primordial gravitational-wave density using recent cosmological data and its impact on cosmic string models, Classical Quantum Gravity 32, 045003 (2015).

[41] R. M. Shannon et al., Gravitational-wave limits from pulsar timing constrain supermassive black hole evolution, Science 342, 334 (2013).

[42] B. Allen and A. C. Ottewill, Detection of anisotropies in the gravitational wave stochastic background, Phys. Rev. D 56, 545 (1997).

[43] N. J. Cornish, Mapping the gravitational wave background, Classical Quantum Gravity 18, 4277 (2001).

[44] S. Mitra, S. Dhurandhar, T. Souradeep, A. Lazzarini, V. Mandic, S. Bose, and S. Ballmer, Gravitational wave radiometry: Mapping a stochastic gravitational wave background, Phys. Rev. D 77, 042002 (2008).

[45] E. Thrane, S. Ballmer, J. D. Romano, S. Mitra, D. Talukder, S. Bose, and V. Mandic, Probing the anisotropies of a stochastic gravitational-wave background using a network of ground-based laser interferometers, Phys. Rev. D 80, 122002 (2009).

[46] J. D. Romano, S. R. Taylor, N. J. Cornish, J. Gair, C. M. F. Mingarelli, and R. van Haasteren, Phase-coherent mapping 
of gravitational-wave backgrounds using ground-based laser interferometers, Phys. Rev. D 92, 042003 (2015).

[47] J. D. Romano and N. J. Cornish, Detection methods for stochastic gravitational-wave backgrounds: A unified treatment, Living Rev. Relativity 20, 2 (2017).

[48] B. P. Abbott et al. (Virgo and LIGO Scientific Collaborations), Directional Limits on Persistent Gravitational Waves from Advanced LIGO?s First Observing Run, Phys. Rev. Lett. 118, 121102 (2017).

[49] C. M. F. Mingarelli, T. Sidery, I. Mandel, and A. Vecchio, Characterizing gravitational wave stochastic background anisotropy with pulsar timing arrays, Phys. Rev. D 88, 062005 (2013).

[50] S. R. Taylor and J.R. Gair, Searching for anisotropic gravitational-wave backgrounds using pulsar timing arrays, Phys. Rev. D 88, 084001 (2013).

[51] J. Gair, J. D. Romano, S. Taylor, and C. M. F. Mingarelli, Mapping gravitational-wave backgrounds using methods from CMB analysis: Application to pulsar timing arrays, Phys. Rev. D 90, 082001 (2014).

[52] C. J. Moore, R. H. Cole, and C. P. L. Berry, Gravitationalwave sensitivity curves, Classical Quantum Gravity 32, 015014 (2015).

[53] B. P. Abbott et al. (LIGO Scientific Collaboration), Exploring the sensitivity of next generation gravitational wave detectors, Classical Quantum Gravity 34, 044001 (2017).

[54] T. Regimbau, The astrophysical gravitational wave stochastic background, Res. Astron. Astrophys. 11, 369 (2011).

[55] G. Cusin, C. Pitrou, and J.-P. Uzan, Anisotropy of the astrophysical gravitational wave background: Analytic expression of the angular power spectrum and correlation with cosmological observations, Phys. Rev. D 96, 103019 (2017).

[56] G. Cusin, C. Pitrou, and J.-P. Uzan, The signal of the gravitational wave background and the angular correlation of its energy density, Phys. Rev. D 97, 123527 (2018).

[57] G. Cusin, I. Dvorkin, C. Pitrou, and J.-P. Uzan, First Predictions of the Angular Power Spectrum of the Astrophysical Gravitational Wave Background, Phys. Rev. Lett. 120, 231101 (2018).

[58] M. Geller, A. Hook, R. Sundrum, and Y. Tsai, Primordial Anisotropies in the Gravitational Wave Background from Cosmological Phase Transitions, Phys. Rev. Lett. 121, 201303 (2018).

[59] S. R. Taylor et al., Limits on Anisotropy in the Nanohertz Stochastic Gravitational-Wave Background, Phys. Rev. Lett. 115, 041101 (2015).

[60] A. Sesana, A. Vecchio, and C. N. Colacino, The stochastic gravitational-wave background from massive black hole binary systems: Implications for observations with pulsar timing arrays, Mon. Not. R. Astron. Soc. 390, 192 (2008).

[61] B. P. Abbott et al. (Virgo and LIGO Scientific Collaborations), Upper Limits on the Stochastic Gravitational-Wave Background from Advanced LIGO First Observing Run, Phys. Rev. Lett. 118, 121101 (2017); Erratum, Phys. Rev. Lett. 119, 029901(E) (2017).

[62] C. Bonvin and R. Durrer, What galaxy surveys really measure, Phys. Rev. D 84, 063505 (2011).
[63] M. Maggiore, Gravitational Waves. Vol. 2: Astrophysics and Cosmology (Oxford University Press, Oxford, 2018).

[64] R. Takahashi and T. Nakamura, Wave effects in the gravitational lensing of gravitational waves from chirping binaries, Astrophys. J. 595, 1039 (2003).

[65] J. A. H. Futterman, F. A. Handler, and R. A. Matzner, Scattering from Black Holes (Cambridge University Press, Cambridge, England, 2009).

[66] R. A. Matzner and M. P. Ryan, Low frequency limit of gravitational scattering, Phys. Rev. D 16, 1636 (1977).

[67] P. J. Westervelt, Scattering of electromagnetic and gravitational waves by a static gravitational field-comparison between the classical (general-relativistic) and quantum field-theoretic results, Phys. Rev. D 3, 2319 (1971).

[68] P. C. Peters, Differential cross-sections for weak field gravitational scattering, Phys. Rev. D 13, 775 (1976).

[69] N.G. Sanchez, Scattering of scalar waves from a Schwarzschild black hole, J. Math. Phys. (N.Y.) 17, 688 (1976).

[70] W. K. De Logi and S. J. Kovacs, Gravitational scattering of zero rest mass plane waves, Phys. Rev. D 16, 237 (1977).

[71] C. Doran and A. Lasenby, Perturbation theory calculation of the black hole elastic scattering cross-section, Phys. Rev. D 66, 024006 (2002).

[72] B. R. Holstein, Graviton physics, Am. J. Phys. 74, 1002 (2006).

[73] S. R. Dolan, Scattering of long-wavelength gravitational waves, Phys. Rev. D 77, 044004 (2008).

[74] E. Guadagnini, Gravitons scattering from classical matter, Classical Quantum Gravity 25, 095012 (2008).

[75] N. Seto and A. Taruya, Polarization analysis of gravitationalwave backgrounds from the correlation signals of groundbased interferometers: Measuring a circular-polarization mode, Phys. Rev. D 77, 103001 (2008).

[76] G. Gubitosi and J. Magueijo, Correlation between oppositehelicity gravitons: Imprints on gravity-wave and microwave backgrounds, Phys. Rev. D 95, 023520 (2017).

[77] R. Kato and J. Soda, Probing circular polarization in stochastic gravitational wave background with pulsar timing arrays, Phys. Rev. D 93, 062003 (2016).

[78] R. Durrer, The Cosmic Microwave Background (Cambridge University Press, Cambridge, England, 2008).

[79] M. Maggiore, Gravitational Waves. Vol. 1: Theory and Experiments, Oxford Master Series in Physics (Oxford University Press, Oxford, 2007).

[80] F. R. N. Schneider et al., The VLT-FLAMES Tarantula survey. XXIX. Massive star formation in the local 30 Doradus starburst, Astron. Astrophys. 618, A73 (2018).

[81] M. V. Zombeck, Handbook of Space Astronomy and Astrophysics, 2nd ed. (Cambridge University Press, Cambridge, England, 1990).

[82] G. Torres, J. Andersen, and A. Giménez, Accurate masses and radii of normal stars: Modern results and applications, Astron. Astrophys. Rev. 18, 67 (2010).

[83] S. Chandrasekhar, The highly collapsed configurations of a stellar mass (Second paper), Mon. Not. R. Astron. Soc. 95, 207 (1935).

[84] D. Koester and G. Chanmugam, Review: Physics of white dwarf stars, Rep. Prog. Phys. 53, 837 (1990). 
[85] G. Meynet, V. Chomienne, S. Ekström, C. Georgy, A. Granada, J. Groh, A. Maeder, P. Eggenberger, E. Levesque, and P. Massey, Impact of mass-loss on the evolution and pre-supernova properties of red supergiants, Astron. Astrophys. 575, A60 (2015).

[86] M. Sasaki, T. Suyama, T. Tanaka, and S. Yokoyama, Primordial black holes: Perspectives in gravitational wave astronomy, Classical Quantum Gravity 35, 063001 (2018).

[87] J. B. Holberg, T. D. Oswalt, and E. M. Sion, A determination of the local density of white dwarf stars, Astrophys. J. 571, 512 (2002).

[88] J. Bovy, Stellar inventory of the solar neighbourhood using Gaia DR1, Mon. Not. R. Astron. Soc. 470, 1360 (2017).

[89] P. Madau and M. Dickinson, Cosmic star formation history, Annu. Rev. Astron. Astrophys. 52, 415 (2014).

[90] G. Cusin, I. Dvorkin, C. Pitrou, and J.-P. Uzan (in preparation).

[91] M. Zumalacarregui and U. Seljak, No LIGO MACHO: Primordial Black Holes, Dark Matter and Gravitational Lensing of Type Ia Supernovae, Phys. Rev. Lett. 121, 141101 (2018).

[92] J. Garcia-Bellido, S. Clesse, and P. Fleury, Primordial black holes survive SN lensing constraints, Phys. Dark Universe 20, 95 (2018).

[93] P. Tisserand et al. (EROS-2 Collaboration), Limits on the macho content of the Galactic Halo from the EROS-2 survey of the magellanic clouds, Astron. Astrophys. 469, 387 (2007).

[94] M. Ricotti, J.P. Ostriker, and K. J. Mack, Effect of primordial black holes on the cosmic microwave background and cosmological parameter estimates, Astrophys. J. 680, 829 (2008).

[95] M. R. S. Hawkins, The case for primordial black holes as dark matter, Mon. Not. R. Astron. Soc. 415, 2744 (2011).
[96] A. M. Green, Astrophysical uncertainties on stellar microlensing constraints on multi-Solar mass primordial black hole dark matter, Phys. Rev. D 96, 043020 (2017).

[97] J. Garca-Bellido and S. Clesse, Constraints from microlensing experiments on clustered primordial black holes, Phys. Dark Universe 19, 144 (2018).

[98] V. Poulin, P. D. Serpico, F. Calore, S. Clesse, and K. Kohri, CMB bounds on disk-accreting massive primordial black holes, Phys. Rev. D 96, 083524 (2017).

[99] Y. Ali-Hamoud and M. Kamionkowski, Cosmic microwave background limits on accreting primordial black holes, Phys. Rev. D 95, 043534 (2017).

[100] A. I. Renzini and C. R. Contaldi, Mapping incoherent gravitational wave backgrounds, Mon. Not. R. Astron. Soc. 481, 4650 (2018).

[101] C. R. Contaldi, Anisotropies of gravitational wave backgrounds: A line of sight approach, Phys. Lett. B 771, 9 (2017).

[102] G. Cusin, C. Pitrou, and J.-P. Uzan, Are we living near the center of a local void?, J. Cosmol. Astropart. Phys. 03 (2017) 038.

[103] P. F. de Salas and S. Pastor, Relic neutrino decoupling with flavour oscillations revisited, J. Cosmol. Astropart. Phys. 07 (2016) 051.

[104] P. A. R. Ade et al. (Planck Collaboration), Planck 2015 results. XIII. Cosmological parameters, Astron. Astrophys. 594, A13 (2016).

[105] F. A. Marin et al. (WiggleZ Collaboration), The WiggleZ dark energy survey: Constraining galaxy bias and cosmic growth with 3-point correlation functions, Mon. Not. R. Astron. Soc. 432, 2654 (2013).

[106] A. Rassat, A. Amara, L. Amendola, F. J. Castander, T. Kitching, M. Kunz, A. Refregier, Y. Wang, and J. Weller, Deconstructing baryon acoustic oscillations: A comparison of methods, arXiv:0810.0003. 